\title{
Isovariant extensors and the characterization of equivariant homotopy equivalences
}

\author{
S. M. Ageev
}

\begin{abstract}
We extend the well-known theorem of James-Segal to the case of an arbitrary family $\mathcal{F}$ of conjugacy classes of closed subgroups of a compact Lie group $G$ : a $G$-map $f: \mathbb{X} \rightarrow \mathbb{Y}$ of metric Equiv ${ }_{\mathcal{F}}$-ANE-spaces is a $G$-homotopy equivalence if and only if it is a weak $G$ - $\mathcal{F}$-homotopy equivalence. The proof is based on the theory of isovariant extensors, which is developed in this paper and enables us to endow $\mathcal{F}$-classifying $G$-spaces with an additional structure.
\end{abstract}

Keywords: classifying $G$-spaces, isovariant absolute extensor, weak equivariant homotopy equivalence.

\section{$\S 1$. Introduction}

Let $G$ be a compact Lie group. We fix a family $\mathcal{F}$ of orbit types (which may be regarded as a subset of the set $\operatorname{Conj}_{G}$ of conjugacy classes of closed subgroups of $G$ ) and consider the category EQUIV $\mathcal{F}^{-T O P}$ of $G$ - $\mathcal{F}$-spaces (that is, $G$-spaces of orbit type $\mathcal{F}$ ). We shall study the identity problem for the classes of $G$-homotopy and

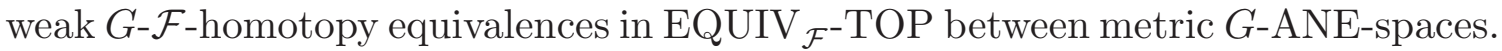
The well-known theorem of James-Segal [1], [2] solves this problem for the maximal family $\mathcal{F}=\operatorname{Conj}_{G}$ and may be used in the case when $\mathcal{F}$ is closed under intersections. However, neither the method used by James-Segal nor other known methods can give anything more since all their constructions are in poor agreement with orbit types.

We note that interest in these questions is primarily motivated by the possibility of sending every $G$-space $\mathbb{X}$ functorially to a $G$-CW-complex $S(\mathbb{X})=S_{\mathcal{F}}(\mathbb{X})$ of orbit type $\mathcal{F}\left(S(\mathbb{X}) \in G_{\mathcal{F}}\right.$-CW for short) which is weakly $G$ - $\mathcal{F}$-homotopy equivalent to $\mathbb{X}$. More precisely, one can find a functor $S_{\mathcal{F}}$ from the category EQUIV-TOP to $G_{\mathcal{F}}-\mathrm{CW}$ and a natural transformation $\mathcal{P}: S_{\mathcal{F}} \rightarrow$ Id such that $\mathcal{P}_{\mathbb{X}}: S_{\mathcal{F}}(\mathbb{X}) \rightarrow \mathbb{X}$ is a weak $G$ - $\mathcal{F}$-homotopy equivalence for every $\mathbb{X}[3]$, [4]. (It should be noted that [3] mentions a gap in [4] and gives rather general hints as to how to fill it.) The orbit space $S_{\mathcal{F}}(\mathbb{X}) / G$, being a $C W$-complex, can be regarded as a homotopy-theoretical orbit space of $\mathbb{X}$ induced by $\mathcal{F}$. Its cohomology is an important equivariant homotopy invariant of $\mathbb{X}$ and generalizes Borel's well-known construction corresponding to the case when $\mathcal{F}$ is a single-element family (see [5], [6]).

This paper was written with the partial support of a grant from the Ministry of Education of the Republic of Belarus.

AMS 2010 Mathematics Subject Classification. 54H15, 54E40, 57S10. 
The significance of this construction is highlighted by the fact that $S_{\mathcal{F}}(*)$, where * means a single-point space, is an $\mathcal{F}$-classifying $G$-space in the sense of [7], [8] (that is, for every $G$-space $\mathbb{Z}$ of orbit type $\mathcal{F}$ there is a $G$-map $f: \mathbb{Z} \rightarrow S_{\mathcal{F}}(*)$ which is unique up to $G$-homotopy). Bredon's equivariant cohomology of $S_{\mathcal{F}}(*)$ may naturally be regarded as generalized cohomology of $G$ (see [9]-[11]).

Despite its significance, this construction does not lead to effective calculations for non-trivial families $\mathcal{F}$. In a series of papers we will develop a new approach based on the theory of isovariant extensors in order to remedy this deficiency. It turns out that $\mathcal{F}$-classifying $G$-spaces can be endowed with an additional structure of isovariant absolute extensors. As a result, we observe an effect of concentration of $\mathcal{F}$-classifying $G$-spaces: all such spaces are realized as $\mathcal{F}$-orbit bundles of the same isovariant absolute extensor. This observation opens up new possibilities for the calculation of homotopy invariants of orbit spaces of $\mathcal{F}$-classifying $G$-spaces. It also yields important information on generalized cohomology of compact groups, including its explicit calculation in particular cases. We shall develop the necessary technique and use it to establish an analogue of the James-Segal theorem in maximal generality.

In what follows we consider only the part of EQUIV $\mathcal{F}^{-T O P}$ consisting of metric $G$ - $\mathcal{F}$-spaces but preserve the same notation for it. We denote the class of injective objects in the category $\mathrm{EQUIV}_{\mathcal{F}^{-}}$TOP by Equiv $\mathcal{F}^{-\mathrm{A}}[\mathrm{N}] \mathrm{E}$. The class Equiv $_{\mathcal{F}}$-AE is always non-empty by Theorems 3.1, 3.3, and it was established in [12] that the class of Equiv-ANE-spaces having orbit type $\mathcal{F}$ coincides with the class of Equiv $\mathcal{F}_{\mathcal{F}}$ ANE-spaces (however, we shall not use this fact). Our generalization of the James-Segal theorem is the following characterization of the weak $G$ - $\mathcal{F}$-homotopy equivalence of Equiv $_{\mathcal{F}}$-ANE-spaces.

Theorem 1.1. Let $\mathbb{X}, \mathbb{Y}$ be Equiv ${ }_{\mathcal{F}}$-ANE-spaces in the category EQUIV F $_{\mathcal{F}}$-TOP. Then a $G$-map $f: \mathbb{X} \rightarrow \mathbb{Y}$ is a weak $G$-F-homotopy equivalence ${ }^{1}$ if and only if $f$ is a G-homotopy equivalence.

Remark 1.2. Since every $G$-homotopy equivalence is easily seen to be a weak $G$-homotopy equivalence, we need only prove the necessity part of Theorem 1.1.

We list some corollaries of Theorem 1.1. First, we characterize the weak $G-\mathcal{F}$ homotopy equivalence for arbitrary orbit types of $\mathbb{X}$ and $\mathbb{Y}$.

Theorem 1.3. Let $\mathbb{X}, \mathbb{Y}$ be Equiv-ANE-spaces. Then a $G$-map $f: \mathbb{X} \rightarrow \mathbb{Y}$ is a weak $G$-F-homotopy equivalence if and only if $f$ is $G$ - $\mathcal{F}$-homotopy soft.

Theorem 1.3 also has several corollaries. We define the $\mathcal{F}$-equivariant envelope of an Equiv-ANE-space $\mathbb{Z}$ to be the set of all $G$-spaces $\mathbb{X} \in$ Equiv $_{\mathcal{F}^{-A N E}}$ admitting a weak $G$-F $\mathcal{F}$-homotopy equivalence $f: \mathbb{X} \rightarrow \mathbb{Z}$. It turns out that the $\mathcal{F}$-homotopy envelope of $\mathbb{Z}$ is non-empty (see Theorems $6.3,6.5$ ), and its equivariant homotopy type is uniquely determined in view of the following fact.

\footnotetext{
${ }^{1}$ That is, the map $f^{H}: \mathbb{X}^{H} \rightarrow \mathbb{Y}^{H}$ of $H$-fixed point sets is a homotopy equivalence for each $(H) \in \mathcal{F}$ and, moreover, $\mathbb{X}^{H} \neq \varnothing$ if and only if $\mathbb{Y}^{H} \neq \varnothing$.
} 
Theorem 1.4. Suppose that $\mathbb{X}, \mathbb{Y} \in$ Equiv $_{\mathcal{F}}$-ANE, $\mathbb{Z} \in$ Equiv-ANE, and $f: \mathbb{X} \rightarrow \mathbb{Z}$ and $g: \mathbb{Y} \rightarrow \mathbb{Z}$ are weak $G$-F-homotopy equivalences. Then there is a $G$-homotopy equivalence $h: \mathbb{X} \rightarrow \mathbb{Y}$ such that $g \circ h \simeq_{G} f$.

We shall deduce this result from Theorem 1.3, although it can also be deduced from the following assertion, which will be proved elsewhere.

Theorem 1.5. Every Equiv $_{\mathcal{F}}$-ANE-space has the G-homotopy type of a $G$-CWcomplex of orbit type $\mathcal{F}$.

It is easy to see that the following three classes of $G$-spaces form a strictly increasing sequence (with respect to inclusion): the class of Equiv ${ }_{\mathcal{F}}$-AE-spaces, the class of $\mathcal{F}$-classifying $G$-spaces, and the class of $G$ - $\mathcal{F}$-spaces whose $H$-fixed-point sets are contractible for all $(H) \in \mathcal{F}$. However, the intersections of these classes with the class of Equiv-ANE-spaces form an identity sequence.

Theorem 1.6. Suppose that the $G-\mathcal{F}$-space $\mathbb{X}$ is an Equiv-ANE-space and $\mathbb{X}^{H}$ is contractible for every $(H) \in \mathcal{F}$. Then $\mathbb{X} \in$ Equiv $_{\mathcal{F}}$-AE.

This theorem has a corollary concerning arbitrary $G$-spaces $\mathbb{X}$. We denote the family $\left\{(H) \mid \mathbb{X}^{H}\right.$ is contractible $\} \subset \operatorname{Orb}(\mathbb{X})$ by $\mathcal{C}_{\mathbb{X}}$.

Theorem 1.7. If $\mathbb{X}$ is an Equiv-ANE-space, then every partial $G$-map $\mathbb{Z} \hookleftarrow$ $\mathbb{A} \stackrel{\varphi}{\longrightarrow} \mathbb{X}, \operatorname{Orb}(\mathbb{Z}) \subset \mathcal{C}_{\mathbb{X}}$, admits a G-extension $\widehat{\varphi}: \mathbb{Z} \rightarrow \mathbb{X}$.

There are several proofs of the James-Segal theorem for the maximal family $\mathcal{F}=$ $\operatorname{Conj}_{G}$. One of them proceeds by finding a $G$-CW-complex that $G$-dominates the original $G$-space [13], and another replaces the original equivariant map $f$ by a map of its cocylinder, which is $G$-homotopy equivalent to $f$ and possesses a number of other useful properties [2]. (Following [6] and [10], we call the resulting functor the equivariant functor of gammafication.) However, both of these proofs operate with intersections of orbit types of points of the original spaces. In the situation of Theorem 1.1, this essentially increases $\mathcal{F}$ and invalidates the known arguments.

To give an example of such difficulties, we note that the equivariant functor of gammafication essentially improves the extensor properties of $G$ - $\mathcal{F}$-spaces and $G$-maps and preserves their equivariant homotopy classes. Such a transformation makes the map $f$ in Theorem 1.1 locally Equiv $_{\mathcal{F}}$-soft and weakly Equiv $_{\mathcal{F}^{-}}$-soft. Then we might hope to complete the proof of Theorem 1.1 using the following theorem, which extends Proposition 4.1 in [2] to the case of $G$ - $\mathcal{F}$-spaces. However, $G$ - $\mathcal{F}$-spaces are not preserved under the equivariant functor of gammafication.

Theorem 1.8. If an equivariant map $f: \mathbb{X} \rightarrow \mathbb{Y}$ between $G$ - $\mathcal{F}$-spaces is locally Equiv $_{\mathcal{F}}$-soft ${ }^{2}$ and weakly Equiv $_{\mathcal{F}}$-soft at the same time, then $f$ is Equiv $_{\mathcal{F}}$-soft and, therefore, is a G-homotopy equivalence.

We overcome all these difficulties by using a new approach which is based on transferring the problem to the category ISOV-TOP whose objects and morphisms are metric $G$-spaces and their isovariant maps.

\footnotetext{
${ }^{2}$ In [2] the term ' $G$-ANE-space over $\mathbb{Y}$ ' was used instead of local Equiv-softness. This indicates an intimate connection between the theories of absolute extensors and soft maps.
} 
The first (and principal) step in the proof of Theorem 1.1 is to study the injective objects in this category, isovariant absolute extensors (or Isov-AE-spaces). Their importance stems from treating the orbit projections of objects in ISOV-TOP (resp. those of Isov-AE-spaces) as generalized principal $G$-bundles (resp. universal generalized principal $G$-bundles) in the sense of Palais [14], §2.6. We mention a result in [15] saying that the orbit space $E$ of any Isov-AE-space $\mathbb{E}$ classifies $G$-spaces in the sense of Palais [14]. Thus the category ISOV-TOP admits a homotopy representation that reduces many aspects of its study to the homotopical properties of topological spaces.

Since ISOV-TOP is a natural extension of the category of principal $G$-bundles, it is equally natural (and useful) to find analogues of other notions and facts in the theory of principal $G$-bundles, such as universal bundles, characteristic classes and so on. However, even the existence of Isov-AE-spaces has so far been known only under certain restrictions on the dimension and orbit type [14], [16], [17]. The following theorem is a definitive improvement of this situation.

Theorem 1.9. Suppose that every $\mathbb{X}_{i}, i \geqslant 1$, is an Isov-generating Equiv-AE-space. Then $\prod\left\{\mathbb{X}_{i} \mid i \geqslant 1\right\} \in$ Isov-AE.

A space $\mathbb{X}$ is said to be Isov-generating if for every metric $G$-space $\mathbb{Z}$ there is an isovariant map $\eta: \mathbb{Z} \rightarrow \mathbb{X}$. Let Con $\mathbb{T}$ be the metric cone over the discrete union $\mathbb{T}$ of all homogeneous spaces $G / H \in G$-ANE. Then the countable power $\mathbb{J} \rightleftharpoons(\text { Con } \mathbb{T})^{\omega}$ is Isov-generating [18]. Since $\mathbb{J}$ is also an Equiv-AE-space and $\mathbb{J} \cong \mathbb{J}^{\omega}$, it follows from Theorem 1.9 that $\mathbb{J} \in$ Isov-AE.

The second important step in the proof of Theorem 1.1 is to transform the equivariant theory to the isovariant one. This is done by introducing Borel's functor. We fix an Isov-AE-space $\mathbb{W}$ and associate every $G$-space $\mathbb{X}$ with the $G$ - $\mathcal{F}$-subspace $E_{\mathcal{F}}(\mathbb{X}) \rightleftharpoons\left\{(w, x) \mid G_{w} \subset G_{x},\left(G_{w}\right) \in \mathcal{F}\right\} \subset \mathbb{W} \times \mathbb{X}$, and every $G$-map $f: \mathbb{X} \rightarrow \mathbb{Y}$ with an isovariant map $E_{f}: E_{\mathcal{F}}(\mathbb{X}) \rightarrow E_{\mathcal{F}}(\mathbb{Y})$ defined by the formula $E_{f}(w, x)=$ $(w, f(x))$. The resulting functor $E_{\mathcal{F}}$ from the equivariant category to the isovariant one is called the Borel functor induced by $\mathcal{F}$.

Note that the Borel functor transforms equivariant homotopy to isovariant homotopy and, therefore, may be regarded as a functor from EQUIV $\mathcal{F}_{\mathcal{F}}-\mathrm{HOMOT}$ to $\mathrm{ISOV}_{\mathcal{F}}$-HOMOT. We shall prove in a future paper that this functor establishes an equivalence between the equivariant homotopy category of Equiv-ANE-spaces and the isovariant homotopy category of Isov-ANE-spaces. This reduces the study of the $G$-homotopy type of Equiv-ANE-spaces to that of the isovariant homotopy type of Isov-ANE-spaces. We can also obtain new information on the equivariant homotopy type of Equiv-ANE-spaces by a deeper investigation of Isov-ANE-spaces.

The following observations describe the main steps of the transformation of the equivariant problem into an isovariant one and thus provide a key to the proof of Theorem 1.1. If $\mathbb{X} \in$ Equiv $_{\mathcal{F}}-\mathrm{ANE}$, then $E_{\mathcal{F}}(\mathbb{X}) \in \operatorname{Isov}_{\mathcal{F}}$-ANE (see property $(*$ ) in $\S 6)$. The $G$-map $p_{\mathbb{X}}: E_{\mathcal{F}}(\mathbb{X}) \rightarrow \mathbb{X}, p_{\mathbb{X}}(w, x)=x$, is a $G$-homotopy equivalence (see Theorem 6.5 ), whence $p_{\mathbb{X}}$ is a weak $G$-homotopy. We similarly have $E_{\mathcal{F}}(\mathbb{Y}) \in$ Isov $\mathcal{F}-\mathrm{ANE}$ and $p_{\mathbb{Y}}: E_{\mathcal{F}}(\mathbb{Y}) \rightarrow \mathbb{Y}$ is a $G$-homotopy equivalence in the case when $\mathbb{Y} \in$ Equiv $_{\mathcal{F}^{-A N E}}$. Since the isovariant map $E_{f}: E_{\mathcal{F}}(\mathbb{X}) \rightarrow E_{\mathcal{F}}(\mathbb{Y})$ makes the 
corresponding diagram commutative (that is, $f \circ p_{\mathbb{X}}=p_{\mathbb{Y}} \circ E_{f}$ ), Theorem 1.1 reduces to a particular case involving isovariant maps.

Theorem 1.10. Suppose that $\mathbb{X}, \mathbb{Y} \in \mathrm{Isov}_{\mathcal{F}}$-ANE. Then an isovariant map $f$ : $\mathbb{X} \rightarrow \mathbb{Y}$ is a $G$-homotopy equivalence if and only if $f$ is a weak $G$-F-homotopy equivalence.

Since the problem of approximating isovariant extensors by simpler objects (such as $G$-CW-complexes) is unsolved in ISOV-TOP, we use other approaches available in the isovariant category. The homotopy density of the embeddings $\mathbb{X}_{H} \hookrightarrow \mathbb{X}^{H}$

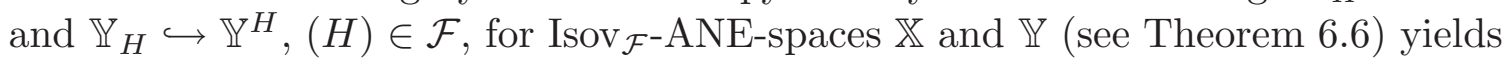
that the notions of weak $G$ - $\mathcal{F}$-homotopy equivalence and weak Isov $\mathcal{F}$-homotopy equivalence coincide for an isovariant map $f: \mathbb{X} \rightarrow \mathbb{Y} .^{3}$ This reduces Theorem 1.10 to the following assertion.

Theorem 1.11. Suppose that $\mathbb{X}, \mathbb{Y} \in \operatorname{Isov}_{\mathcal{F}}$-ANE. Then an isovariant map $f$ : $\mathbb{X} \rightarrow \mathbb{Y}$ is an Isov-homotopy equivalence if and only if $f$ is a weak Isov $_{\mathcal{F}}$-homotopy equivalence. ${ }^{4}$

We shall prove Theorem 1.11 using an appropriate functor of isovariant gammafication which is free from the defects of its equivariant analogue. It turns out that after such a transformation the isovariant map $f$ in Theorem 1.11 preserves the isovariant homotopy class and satisfies the hypotheses of the following theorem.

Theorem 1.12. Let $f: \mathbb{X} \rightarrow \mathbb{Y}$ be an isovariant map between Isov $_{\mathcal{F}}$-ANE-spaces. Suppose that $f$ is locally $\operatorname{Isov}_{\mathcal{F}}$-soft and weakly $\operatorname{Isov}_{\mathcal{F}}$-soft. ${ }^{5}$ Then $f$ is $\operatorname{Isov}_{\mathcal{F}}$-soft.

Since an $\operatorname{Isov}_{\mathcal{F}}$-soft map is an Isov-homotopy equivalence, this proves Theorem 1.11 and hence Theorems 1.10, 1.1. In $\S 10$ we state some problems and conjectures related to the contents of this paper.

\section{$\S 2$. Preliminaries}

Throughout the paper, all spaces are assumed to be metric and all maps continuous unless they are defined by certain constructions or unless otherwise stated. All group actions are actions of compact Lie groups.

We describe the key notions of the theory of $G$-spaces [20]. An action of a compact group $G$ on a space $\mathbb{X}$ is a continuous map $\mu$ from the product $G \times \mathbb{X}$ to $\mathbb{X}$ with the following properties:

1) $\mu(g, \mu(h, x))=\mu(g \cdot h, x)$,

2) $\mu(e, x)=x$ for all $x \in \mathbb{X}, g, h \in G$ (here $e$ is the identity of $G$ ).

As a rule, we write $g \cdot x$ or simply $g x$ instead of $\mu(g, x)$. A space $\mathbb{X}$ with an action of $G$ is called a $G$-space. A map $f: \mathbb{X} \rightarrow \mathbb{Y}$ of $G$-spaces is called a $G$-map or

\footnotetext{
${ }^{3}$ This is an infinite-dimensional analogue of the so-called 'large gap' condition [19] which enables us to transform homotopically an equivariant homotopy equivalence between manifolds into an isovariant one.

${ }^{4}$ That is, the map $f_{H}: \mathbb{X}_{H} \rightarrow \mathbb{Y}_{H}$ is a homotopy equivalence for each $(H) \in \mathcal{F}$ and, in addition, $\mathbb{X}_{H} \neq \varnothing$ if and only if $\mathbb{Y}_{H} \neq \varnothing$.

${ }^{5}$ We say that an isovariant $G$-map $f: \mathbb{X} \rightarrow \mathbb{Y}$ is weakly Isov $\mathcal{F}$-soft if $f_{H}: \mathbb{X}_{H} \rightarrow \mathbb{Y}_{H}$ is soft for each $(H) \in \mathcal{F}, \mathbb{Y}_{H} \neq \varnothing$.
} 
an equivariant map if $f(g \cdot x)=g f(x)$ for all $x \in \mathbb{X}, g \in G$. An equivariant homeomorphism is called an equimorphism.

Note that $G$-spaces and $G$-maps form a category, which is denoted by $G$-TOP (or EQUIV-TOP if $G$ is clear from the context). The equivariant analogue of any notion $*$ of non-equivariant topology will often be denoted by $G$-* or Equiv-*. Conversely, every notion dealing with $G$-spaces yields a non-equivariant notion by taking the group $G$ to be trivial.

The orbit $G(x)$ of a point $x \in \mathbb{X}$ is the subset $\{g \cdot x \mid g \in G\}=G \cdot x$, which turns out to be closed. The natural map $\pi=\pi_{\mathbb{X}}: \mathbb{X} \rightarrow X, x \mapsto G(x)$, from $\mathbb{X}$ to the quotient space is called the orbit projection. The quotient space $X$ equipped with the quotient topology (induced by $\pi$ ) is called the orbit space. Clearly, $G \cdot A=$ $\pi^{-1} \pi(A)$ for every subset $A \subset \mathbb{X}$. If $A \subset \mathbb{X}$ coincides with $G \cdot A$, then it inherits a natural action of $G$ and is called an invariant subset or a $G$-subset.

Every equivariant map $f: \mathbb{X} \rightarrow \mathbb{Y}$ transforms the orbit of a point $x$ to the orbit of $f(x)$. Hence we have an induced map of orbit spaces $\tilde{f}: X \rightarrow Y, \tilde{f}(G(x))=$ $G(f(x))$, which is continuous because orbit projections are quotients and $f$ is continuous.

For every $x \in \mathbb{X}$, the subset $G_{x}=\{g \in G \mid g \cdot x=x\}$ is a closed subgroup of $G$. It is called the stabilizer of $x$. For every closed subgroup $H$ ( $H<G$ for short) we consider the following subsets of $\mathbb{X}$ :

$$
\mathbb{X}^{H}=\{x \in \mathbb{X} \mid H \cdot x=x\}=\left\{x \in \mathbb{X} \mid H \subset G_{x}\right\}
$$

(the set of $H$-fixed points),

$$
\mathbb{X}_{H}=\left\{x \in \mathbb{X} \mid H=G_{x}\right\}, \quad \mathbb{X}_{(H)}=\left\{x \in \mathbb{X} \mid H \text { is conjugate to } G_{x}\right\}
$$

Since an equivariant map $f: \mathbb{X} \rightarrow \mathbb{Y}$ commutes with the group action, we have $f\left(\mathbb{X}^{H}\right) \subset \mathbb{Y}^{H}$. We denote the restriction $f \uparrow: \mathbb{X}^{H} \rightarrow \mathbb{Y}^{H}$ by $f^{H}$.

An equivariant map $f: \mathbb{X} \rightarrow \mathbb{Y}$ is said to be isovariant if it preserves stabilizers, that is, $G_{x}=G_{f(x)}$ for all $x \in \mathbb{X}$. Given an isovariant map $f$, we denote the restriction $f \uparrow: \mathbb{X}_{H} \rightarrow \mathbb{Y}_{H}$ by $f_{H}$. The category of $G$-spaces and isovariant maps is denoted by ISOV $_{G^{-}}$TOP (or ISOV-TOP if $G$ is clear from the context). The following equimorphism criterion is well known. An isovariant map is an equimorphism if and only if the induced map of orbits is a homeomorphism (see [20], Ch.1, Exercise 10).

We introduce several concepts related to the extension of $G$-maps in a category $\mathfrak{C}$ which is either ISOV-TOP or EQUIV-TOP. A space $\mathbb{X}$ acted on by a compact group $G$ is called an absolute neighbourhood $\mathfrak{C}$-extensor (and we write $\mathbb{X} \in \mathfrak{C}$-ANE) if every $\mathfrak{C}$-morphism $\varphi: \mathbb{A} \rightarrow \mathbb{X}$ defined on a closed $G$-subset $\mathbb{A} \subset \mathbb{Z}$ of a $G$-space $\mathbb{Z}$ (and called a partial $\mathfrak{C}$-morphism) can be extended to a $\mathfrak{C}$-morphism $\widehat{\varphi}: \mathbb{U} \rightarrow \mathbb{X}$ of some $G$-neighbourhood $\mathbb{U} \subset \mathbb{Z}$ of $\mathbb{A}$. If $\mathbb{U}$ can always be taken equal to $\mathbb{Z}$, then $\mathbb{X}$ is called an absolute $\mathfrak{C}$-extensor, $\mathbb{X} \in \mathfrak{C}$-AE. When $G$ is trivial (that is, we are considering spaces without an action), this notion reduces to that of an absolute [neighbourhood] extensor for metric spaces, $\mathrm{A}[\mathrm{N}] \mathrm{E}[21]$. 
If $\mathfrak{C}$ is the category EQUIV-TOP, then the absolute [neighbourhood] $\mathfrak{C}$-extensors are referred to as equivariant [neighbourhood] extensors (or, briefly, Equiv-A[N]Espaces). If $\mathfrak{C}=$ ISOV-TOP, then the absolute [neighbourhood] $\mathfrak{C}$-extensors are referred to as isovariant [neighbourhood] extensors (or, briefly, Isov-A[N]E-spaces). Any Isov-AE-space is a universal $G$-space in the sense of Palais [14], and its orbit space classifies $G$-spaces.

If two $\mathfrak{C}$-morphisms $f, g: \mathbb{X} \rightarrow \mathbb{Y}$ can be connected by a $\mathfrak{C}$-homotopy $H$ : $\mathbb{X} \times[0,1] \rightarrow \mathbb{Y}$, then we write $f \simeq_{\mathfrak{C}} g$. This notation takes the form $f \simeq_{G} g$ or $f \simeq_{\text {Equiv }} g$ for $\mathfrak{C}=$ EQUIV, and $f \simeq_{\text {Isov }} g$ for $\mathfrak{C}=$ ISOV. We call a $\mathfrak{C}$-map $f: \mathbb{X} \rightarrow \mathbb{Y}$ a $\mathfrak{C}$-homotopy equivalence if there is a $\mathfrak{C}$-map $g: \mathbb{Y} \rightarrow \mathbb{X}$ such that $g \circ f$ and $f \circ g$ are $\mathfrak{C}$-homotopic to $\operatorname{Id}_{\mathbb{X}}$ and $\operatorname{Id}_{\mathbb{Y}}$ respectively. It is easily seen that if $f$ is an Isov-homotopy equivalence (an Equiv-homotopy equivalence), then $f_{H}: \mathbb{X}_{H} \rightarrow \mathbb{Y}_{H}$ $\left(f^{H}: \mathbb{X}^{H} \rightarrow \mathbb{Y}^{H}\right)$ is a homotopy equivalence for every subgroup $H<G$.

Suppose that $\mathcal{F} \subset \operatorname{Conj}_{G}$. Then the set $\mathbb{X}_{\mathcal{F}} \rightleftharpoons\left\{x \mid\left(G_{x}\right) \in \mathcal{F}\right\} \subset \mathbb{X}$ is called the $\mathcal{F}$-orbit bundle of $\mathbb{X}$. We say that a $G$-space $\mathbb{X}$ is of orbit type $\mathcal{F}$ (or is a $G$ - $\mathcal{F}$-space) if $\mathbb{X}=\mathbb{X}_{\mathcal{F}}$. A $G$ - $\mathcal{F}_{\text {-space }} \mathbb{X}$ is called a $\mathfrak{C}_{\mathcal{F}}$-A $[\mathrm{N}] \mathrm{E}$-space if every partial $\mathfrak{C}$-morphism $\varphi: \mathbb{A} \rightarrow \mathbb{X}$ defined on a closed subset of a $G$ - $\mathcal{F}$-space $\mathbb{Z}$ can be extended to a $\mathfrak{C}$-morphism $\widehat{\varphi}: \mathbb{Z} \rightarrow \mathbb{X}$ on the whole of $\mathbb{Z}$ [on a $G$-neighbourhood $\mathbb{U} \subset \mathbb{Z}$ of $\mathbb{A}]$. This yields the definitions of Equiv $_{\mathcal{F}}-\mathrm{A}[\mathrm{N}] \mathrm{E}-$ and $\operatorname{Isov}_{\mathcal{F}}-\mathrm{A}[\mathrm{N}] \mathrm{E}$-spaces. The following theorem on the extension of $\mathfrak{C}$-homotopy is established by a modification of the standard Borsuk procedure.

Theorem 2.1. Suppose that $\mathbb{X} \in \mathfrak{C}_{\mathcal{F}}$-ANE and $H: \mathbb{A} \times[0,1] \rightarrow \mathbb{X}$ is a $\mathfrak{C}$-homotopy connecting partial $\mathfrak{C}$-maps $f, g: \mathbb{A} \rightarrow \mathbb{X}$ such that $f$ extends to a $\mathfrak{C}$-map $\hat{f}: \mathbb{Z} \rightarrow \mathbb{X}$, $\operatorname{Orb}(\mathbb{Z}) \subset \mathcal{F}$. Then the $\mathfrak{C}$-homotopy $H$ extends to a $\mathfrak{C}$-homotopy $\widehat{H}: \mathbb{Z} \times[0,1] \rightarrow \mathbb{X}$ connecting $\hat{f}$ with a $\mathfrak{C}$-map $\hat{g}: \mathbb{Z} \rightarrow \mathbb{X}$.

Proof. We consider an invariant subset $M(\mathbb{X}, \mathbb{A}) \rightleftharpoons(\mathbb{X} \times\{0\}) \cup(\mathbb{A} \times I)$ of the $\mathfrak{C}$-space $\mathbb{X} \times I$. Defining a map $\xi: M(\mathbb{X}, \mathbb{A}) \rightarrow \mathbb{Z}$ by the formula

$$
\xi(x, t)= \begin{cases}f(x) & \text { if } \quad(x, t) \in \mathbb{X} \times\{0\}, \\ H(x, t) & \text { if } \quad(x, t) \in \mathbb{A} \times I,\end{cases}
$$

we easily see that $\xi$ is a continuous $\mathfrak{C}$-map. Since $\mathbb{Z} \in \mathfrak{C}_{\mathcal{F}}$-ANE by hypothesis and $M(\mathbb{X}, \mathbb{A}) \subset \mathbb{X} \times I$ is a closed $G$-subspace of the $\mathfrak{C}$-space $\mathbb{X} \times I$, there is a neighbourhood $\mathbb{V}$ of the set $M(\mathbb{X}, \mathbb{A}) \subset \mathbb{X} \times I$ such that $\xi$ admits a neighbourhood $\mathfrak{C}$-extension $\widehat{\xi}: \mathbb{V} \rightarrow \mathbb{Z}$.

Let $\mathbb{U}$ be an invariant neighbourhood of $\mathbb{A}$ in $\mathbb{X}$ such that $\mathbb{U} \times I \subset \mathbb{V}$, and let $\beta: \mathbb{X} \rightarrow I$ be the Urysohn invariant function such that $\beta \uparrow_{\mathbb{A}} \equiv 1$ and $\beta \uparrow_{\mathbb{X} \backslash \mathbb{U}} \equiv 0$. Defining a map $\alpha: \mathbb{X} \times I \rightarrow \mathbb{X} \times I$ by the formula $\alpha(x, t)=(x, \beta(x) \cdot t)$, we easily see that it is continuous and $\alpha(\mathbb{X} \times I) \subset \mathbb{V}$.

We claim that the formula $\widehat{H}(x, t)=\widehat{\xi}(\alpha(x, t))$ gives the desired $\mathfrak{C}$-homotopy $\widehat{H}: \mathbb{X} \times I \rightarrow \mathbb{Z}$. Indeed, for every $x \in \mathbb{X}$ we have

$$
\widehat{H}_{0}(x)=\widehat{H}(x, 0)=\widehat{\xi}(\alpha(x, 0))=\widehat{\xi}(x, 0)=\xi(x, 0)=f(x) .
$$


Moreover, for every point $(a, t) \in \mathbb{A} \times I$,

$$
\widehat{H}(a, t)=\widehat{\xi}(\alpha(a, t))=\xi(\alpha(a, t))=\xi(a, t)=H(a, t) .
$$

Equivariant homotopy equivalence is closely related to the notion of $G$-homotopy softness. We say that an equivariant map $f: \mathbb{X} \rightarrow \mathbb{Y}$ is $G$-homotopy soft if for every $G$-homotopy commutative diagram

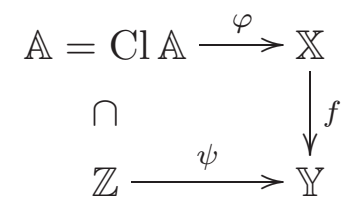

in the category EQUIV-TOP there is a $G$-map $\widehat{\varphi}: \mathbb{Z} \rightarrow \mathbb{X}$ extending $\varphi$ and satisfying $f \circ \widehat{\varphi} \simeq_{G} \psi$. If a $G$-map $\widehat{\varphi}: \mathbb{Z} \rightarrow \mathbb{X}$ with the required properties exists under the additional condition $\operatorname{Orb}(\mathbb{Z}) \subset \mathcal{F}$, where $\mathcal{F} \subset \operatorname{Conj}_{G}$, then we get the notion of a $G$ - $\mathcal{F}$-homotopy soft map.

It is easily checked that every $G$ - $\mathcal{F}$-homotopy soft $G$-map $f: \mathbb{X} \rightarrow \mathbb{Y}$ is a weak $G$ - $\mathcal{F}$-homotopy equivalence. It is also clear that if $f: \mathbb{X} \rightarrow \mathbb{Y}$ is $G$-homotopy soft, then it is an Equiv-homotopy equivalence. The converse holds under the following additional condition.

$(*)$ If $\mathbb{X} \in$ Equiv $_{\mathcal{F}}$-ANE, then every Equiv-homotopy equivalence $f: \mathbb{X} \rightarrow \mathbb{Y}$ is $G$ - $\mathcal{F}$-homotopy soft.

To prove $(*)$, we consider a $G$-map $g: \mathbb{Y} \rightarrow \mathbb{X}$ which is $G$-homotopy inverse to $f$. Since the $G$-maps $\varphi: \mathbb{A} \rightarrow \mathbb{X}$ and $g \circ(f \circ \varphi)=g \circ \psi \uparrow_{\mathbb{A}}: \mathbb{A} \rightarrow \mathbb{X}$ are $G$-homotopic, Theorem 2.1 on the extension of $G$-homotopy yields a $G$-map $\widehat{\varphi}: \mathbb{Z} \rightarrow \mathbb{X}$ which extends $\varphi$ and is $G$-homotopic to $g \circ \psi$. Hence we have $f \circ \widehat{\varphi} \simeq_{G}(f \circ g) \circ \psi \simeq_{G} \psi$.

Combining this with Theorem 2.1, we get the following proposition.

Proposition 2.2. If $\mathbb{X}, \mathbb{Y} \in$ Equiv $_{\mathcal{F}}$-ANE have the same equivariant homotopy type, then $\mathbb{X} \in$ Equiv $_{\mathcal{F}}-\mathrm{AE}$ if and only if $\mathbb{Y} \in$ Equiv $_{\mathcal{F}}-\mathrm{AE}$.

Proof. If $f: \mathbb{X} \rightarrow \mathbb{Y}$ is an Equiv-homotopy equivalence, then $f$ is $G$-homotopy soft by property $(*)$. Therefore given a partial $G$-map $\mathbb{Z} \hookleftarrow \mathbb{A} \stackrel{\varphi}{\rightarrow} \mathbb{Y}, \operatorname{Orb}(\mathbb{Z}) \subset \mathcal{F}$, there is a $G$-map $\psi: \mathbb{Z} \rightarrow \mathbb{X}$ such that $\varphi \simeq_{G} f \circ \psi$. Since $\mathbb{X} \in$ Equiv $_{\mathcal{F}^{-A E}}$, there is a $G$-extension $\widehat{\psi}: \mathbb{Z} \rightarrow \mathbb{X}$ of $\psi$. Hence we have $\varphi \simeq_{G} f \circ \psi$, and $f \circ \psi$ has a $G$-extension $f \circ \widehat{\psi}$ to the whole of $\mathbb{Z}$. By Theorem 2.1, $\varphi$ has a $G$-extension $f \circ \widehat{\psi}$ to the whole of $\mathbb{Z}$.

Let $\mathbb{C} \stackrel{g}{\rightarrow} \mathbb{A} \stackrel{f}{\leftarrow} \mathbb{B}$ be two $G$-maps. We define the fibrewise product of the $G$-spaces $\mathbb{C}$ and $\mathbb{B}$ with respect to $g$ and $f$ to be the $G$-subset $\{(c, b) \mid g(c)=$ $f(b)\} \subset \mathbb{C} \times \mathbb{B}$ and denote it by $\mathbb{C}_{g} \times_{f} \mathbb{B}$. The projections of $\mathbb{D}=\mathbb{C}_{g} \times_{f} \mathbb{B}$ onto the factors $\mathbb{C}$ and $\mathbb{B}$ generate $G$-maps $\check{f}: \mathbb{D} \rightarrow \mathbb{C}$ and $\check{g}: \mathbb{D} \rightarrow \mathbb{B}$. We say that $\check{f}$ (resp. $\check{g}$ ) is parallel to $f$ (resp. $g$ ) and write $\check{f} \| f$ and $\check{g} \| g$. It is known that for any commutative $G$-diagram

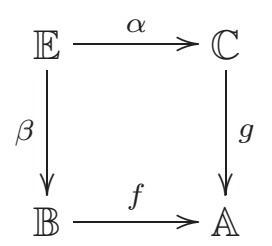


there is a unique $G$-map $h: \mathbb{E} \rightarrow \mathbb{D}$ that splits the corresponding diagram into two commutative triangles, $\check{g} \circ h=\beta$ and $\check{f} \circ h=\alpha$.

Note that the $G$-map $f \circ \check{g}=g \circ \check{f}: \mathbb{D} \rightarrow \mathbb{A}$ is the product of the $G$-maps $g$ and $f$ in the category $G$-TOP $A$ of $G$-spaces over $\mathbb{A}$. The most important examples of fibrewise products in the theory of topological transformation groups are provided by isovariant maps.

Proposition 2.3. Let $h: \mathbb{Y} \rightarrow \mathbb{X}$ be an isovariant map and $\tilde{h}: Y \rightarrow X$ the map of orbit spaces induced by $h$. Then $\mathbb{Y}$ is a fibrewise product $Y_{\tilde{h}} \times_{\pi_{\mathbb{X}}} \mathbb{X}, h$ is parallel to $\tilde{h}$, and the orbit projection $\pi_{\mathbb{Y}}$ is parallel to $\pi_{\mathbb{X}}$ (we regard the orbit spaces as having the trivial action of $G$ ).

Proof. This follows easily from the equimorphism criterion stated above (see [20], Ch. 1, Exercise 10).

\section{$\S 3$. Isovariant absolute extensors}

The isovariant absolute extensors in the class of products of $G$-spaces are described by Theorem 1.9. We shall use the following refined form.

Theorem 3.1. Let $\mathbb{X}_{i}$ be an Isov-generating Equiv-AE-space for every $i \geqslant 1$. Then for every partial $G$-map $\mathbb{Z} \hookleftarrow \mathbb{A} \stackrel{\varphi}{\longrightarrow} \mathbb{X} \rightleftharpoons \prod\left\{\mathbb{X}_{i} \mid i \geqslant 1\right\}$ there is a $G$-map $\psi: \mathbb{Z} \rightarrow \mathbb{X}$ which extends $\varphi$ and is isovariant on the complement (that is, $\psi \Upsilon_{\mathbb{Z} \backslash \mathbb{A}}$ is isovariant).

Proof. Since $\mathbb{A} \subset \mathbb{X}$ is closed, we can choose a sequence of neighbourhoods $\mathbb{Z}=$ $\mathbb{U}_{0} \ni \mathbb{U}_{1} \ni \cdots$ and $G$-functions $\chi_{i}: \mathbb{Z} \rightarrow[0,1], i \geqslant 1$, such that $\bigcap \mathbb{U}_{i}=\mathbb{A}$, $\chi_{i}^{-1}(0) \supset \mathbb{Z} \backslash \mathbb{U}_{i}$ and $\chi_{i}^{-1}(1) \supset \mathbb{U}_{i+1}$. (We recall that an inclusion $A \subset B$ is said to be strong and is written as $A \Subset B$ if $\mathrm{Cl} A \subset \operatorname{Int} B$.)

Since $\mathbb{X} \in$ Equiv-AE, there is an equivariant extension $\widehat{\varphi}: \mathbb{Z} \rightarrow \mathbb{X}$ of the map $\varphi$. Now, starting with $\widehat{\varphi}$, we seek a $G$-map $\psi: \mathbb{Z} \rightarrow \mathbb{X}$ extending $\varphi=\widehat{\varphi} \uparrow_{\mathbb{A}}$ in such a way that $\psi \Upsilon_{\mathbb{Z} \backslash \mathbb{A}}$ is isovariant. We write $\widehat{\varphi}$ in the form $\prod \widehat{\varphi}_{i}$, where each map $\widehat{\varphi}_{i}: \mathbb{Z} \rightarrow \mathbb{X}_{i}$ is equivariant, and fix an isovariant map $e_{i}: \mathbb{Z} \rightarrow \mathbb{X}_{i}$ (which exists since $\mathbb{X}_{i}$ is Isov-generating). Let $H_{i}: \mathbb{Z} \times I \rightarrow \mathbb{X}_{i}$ be an Equiv-homotopy connecting $e_{i}$ with $\widehat{\varphi}_{i}$ (which exists since $\mathbb{X}_{i} \in$ Equiv-AE). Then the desired map $\psi$ is given by the formula

$$
\begin{gathered}
\psi \uparrow_{\mathbb{A}}=\widehat{\varphi} \uparrow_{\mathbb{A}}=\varphi \\
\left(\psi \uparrow_{\mathbb{U}_{i} \backslash \mathbb{U}_{i+1}}\right)(z)=\widehat{\varphi}_{1} \times \cdots \times \widehat{\varphi}_{i-1} \times H_{i}\left(z, \chi_{i}(z)\right) \times e_{i+1} \times \cdots, \quad i \geqslant 0 .
\end{gathered}
$$

It is easy to verify that $\psi$ is continuous and also isovariant on the complement.

We fix a closed topological embedding $j: X \hookrightarrow L$ of the orbit space of an arbitrary $G$-space $\mathbb{X}$ in a normed vector space $L[22]$. Since the countable power $\mathbb{J}$ of the metric cone Con $\mathbb{T}$ over $\mathbb{T}=\sqcup\{G / H \mid G / H \in G$-ANE $\}$ is Isov-generating [18], there is an isovariant map $f: \mathbb{X} \rightarrow \mathbb{J}$. Clearly, the product $(j \circ p) \times f$ is a closed topological $G$-embedding of $\mathbb{X}$ in the $G$-space $\mathbb{Y} \rightleftharpoons L \times \mathbb{J}$, which is easily seen to be Isov-AE. This proves the following result.

Theorem 3.2. Every $G$-space admits a closed $G$-embedding in the Isov-AE-space $L \times \mathbb{J}$. 
Let $\Re$ be the set of all irreducible orthogonal representations of $G$ (including the trivial representation), $\mathbb{R}_{\varrho}$ the space of a representation $\varrho \in \mathfrak{R}$, and $\mathbb{D}_{\varrho}$ its unit ball. By definition, the equivariant Hilbert space $\mathbb{L}_{2}$ is the product

$$
\left\{\left(v_{\varrho}\right) \in\left(\bigoplus_{\varrho \in \mathfrak{R}} \mathbb{R}_{\varrho}\right)^{\omega} \mid \sum\left\|v_{\varrho}\right\|^{2}<\infty\right\},
$$

and the equivariant Hilbert cube $\mathbb{Q}$ (only for compact metric groups $G$ ) is $\left(\prod\left\{\mathbb{D}_{\varrho} \mid\right.\right.$ $\varrho \in \mathfrak{R}\})^{\omega}$.

We note without proof that the equivariant Hilbert space $\mathbb{L}_{2}$ and equivariant Hilbert cube $\mathbb{Q}$ are Isov-AE-spaces. The product of any compact Isov-AE-space $\mathbb{X}$ and the Hilbert cube $Q$ is equimorphic to the equivariant Hilbert cube $\mathbb{Q}$. Another example of an Isov-AE-space is the space $C(G, L)$ (with the uniform metric) of all continuous maps $f: G \rightarrow L$ to the Hilbert space $L$ of weight $w(G)$ endowed with the (continuous) action of the group $G$ by the formula

$$
(g \cdot f)(h)=f\left(g^{-1} \cdot h\right), \quad f \in C(G, L), \quad g, h \in G .
$$

These and other results of the theory of isovariant extensors will be published elsewhere.

By Theorem 3.2, every Isov-A[N]E-space $\mathbb{X}$ admits a closed $G$-embedding in the $G$-space $L \times \mathbb{J}$ satisfying the conclusion of Theorem 3.1. Since $\mathbb{X} \in$ Isov-A[N]E, there is an isovariant retraction $r: L \times \mathbb{J} \rightarrow \mathbb{X}$ [a neighbourhood isovariant retraction $r: \mathbb{U} \rightarrow \mathbb{X}$. Hence Theorem 3.1 yields an important relation between the injective objects in the isovariant and equivariant categories.

Theorem 3.3. Every Isov-A[N]E-space $\mathbb{X}$ satisfies the conclusion of Theorem 3.1 and, therefore, every Isov-A[N]E-space is an Equiv-A[N]E-space.

It is easy to give an example of an Equiv-AE-space $\mathbb{Z} \notin$ Isov-ANE. The following results describe the difference between these two classes. If $\mathbb{X} \in$ Equiv-ANE, then $\left\{\mathbb{X}^{H} \mid H<G\right\} \in$ equi-LAE. If $\mathbb{X} \in$ Isov-ANE, then $\left\{\mathbb{X}_{H} \mid H<G\right\} \in$ equi-LAE. If Con $\mathbb{X} \in$ Isov-AE and $\mathbb{X}^{G} \in \mathrm{AE}$, then $\mathbb{X} \in$ Isov-AE.

\section{$\S$ 4. Soft maps}

Throughout this section, $\mathcal{D}$ stands for a commutative diagram

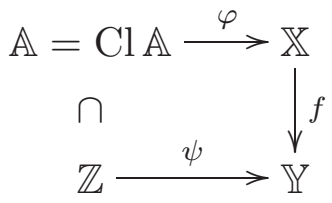

in a category $\mathfrak{C}$ which is either EQUIV-TOP or ISOV-TOP, and $\widetilde{\mathcal{D}}$ stands for the commutative diagram of orbit spaces generated by $\mathcal{D}$.

The morphism $\varphi$ is called a partial lifting of the morphism $\psi$ with respect to $f$. We say that the problem of extending the partial lifting for $\mathcal{D}$ is globally (locally) 
soluble if there is a morphism $\widehat{\varphi}: \mathbb{Z} \rightarrow \mathbb{X}(\widehat{\varphi}: \mathbb{U} \rightarrow \mathbb{X}$, where $\mathbb{U} \subset \mathbb{Z}$ is a neighbourhood of $\mathbb{A}$ ) extending $\varphi$ and satisfying $f \circ \widehat{\varphi}=\psi$ (resp. $f \circ \widehat{\varphi}=\psi \uparrow_{\mathbb{U}}$ ). We also say that $\widehat{\varphi}: \mathbb{Z} \rightarrow \mathbb{X}(\widehat{\varphi}: \mathbb{U} \rightarrow \mathbb{X})$ is a global (local) lifting of $\psi$, or $\widehat{\varphi}$ is a global (local) splitting of the commutative diagram $\mathcal{D}$.

If $\mathbb{Z}=\mathbb{Y}$, then the problem of the global extension of a partial lifting for $\mathcal{D}$ is transformed into the problem of the global extension of a partial section of $f$.

Definition 4.1. Suppose that $\mathcal{F} \subset \operatorname{Conj}_{G}$. A morphism $f: \mathbb{X} \rightarrow \mathbb{Y}$ in the category $\mathfrak{C}$ is said to be (locally) $\mathfrak{C}_{\mathcal{F}}$-soft if for every commutative square diagram $\mathcal{D}$ in $\mathfrak{C}$ with a $G$ - $\mathcal{F}$-space $\mathbb{Z}$, the problem of extending the partial lifting is (locally) globally soluble. ${ }^{6}$

Note that these notions may be interpreted as giving the injective objects of the corresponding category of morphisms. We give the simplest examples of $\mathfrak{C}$-soft maps. If $\mathbb{Z} \in$ Equiv-AE, then pr: $\mathbb{Y} \times \mathbb{Z} \rightarrow \mathbb{Y}$ is an Equiv-soft map, and the isovariant map $\varepsilon \rightleftharpoons \operatorname{pr}\left\lceil: E(\mathbb{Y}, \mathbb{Z}) \rightleftharpoons\left\{(y, z) \mid G_{y} \subset G_{z}\right\} \rightarrow \mathbb{Y}\right.$ is an Isov-soft map.

Another example of a soft morphism arises from the construction of the space of paths $\mathbb{Y}^{I} \rightleftharpoons C([0,1], \mathbb{Y})$. We define a $G$-map $p: \mathbb{Y}^{I} \rightarrow \mathbb{Y}$ by the formula $p(\lambda)=\lambda(0)$. The following fact is an easy reformulation of Theorem 2.1 on the extension of equivariant homotopy. It is proved in the same way as Proposition 7.1.

Proposition 4.2. If $\mathbb{Y}$ is Equiv-ANE, then the map $p: \mathbb{Y}^{I} \rightarrow \mathbb{Y}$ is Equiv-soft.

We easily see that every $\mathfrak{C}$-soft map $f: \mathbb{X} \rightarrow \mathbb{Y}$ is a $G$-homotopy equivalence and a Hurewicz $\mathfrak{C}$-bundle. The latter means that for every commutative square

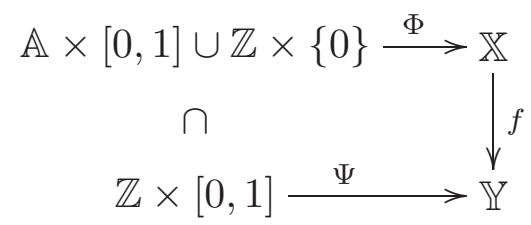

in the category $\mathfrak{C}$, where $\mathbb{A} \subset \mathbb{Z}$ is a closed $\mathfrak{C}$-subspace, there is a $\mathfrak{C}$-map $\widehat{\Phi}$ : $\mathbb{Z} \times[0,1] \rightarrow \mathbb{X}$ extending $\Phi$ and satisfying $f \circ \widehat{\Phi}=\Psi$. The converse also holds.

Theorem 4.3. A map $f: \mathbb{X} \rightarrow \mathbb{Y}$ is $\mathfrak{C}$-soft if and only if $f$ is a Hurewicz $\mathfrak{C}$-bundle and a $\mathfrak{C}$-homotopy equivalence.

Proof. The proof of sufficiency is based on the following two lemmas which are known for trivial actions (see [23]).

Lemma 4.4. If $f: \mathbb{X} \rightarrow \mathbb{Y}$ is a Hurewicz $\mathfrak{C}$-bundle and a $\mathfrak{C}$-homotopy equivalence, then $f$ is fibrewise $\mathfrak{C}$-contractible, that is, there exists a $\mathfrak{C}$-homotopy $H: \mathbb{X} \times I \rightarrow \mathbb{X}$ such that $H_{0}=\mathrm{Id}, f \circ H_{t}=f$ and $H_{1}=s \circ f$ for some $\mathfrak{C}$-section $s: \mathbb{Y} \rightarrow \mathbb{X}$.

Proof. Let $g: \mathbb{Y} \rightarrow \mathbb{X}$ be a $\mathfrak{C}$-map such that $f \circ g \stackrel{F}{\sim_{\mathfrak{C}}} \operatorname{Id}_{\mathbb{Y}}$ and $\operatorname{Id}_{\mathbb{X}} \stackrel{H_{\mathfrak{C}}}{\simeq_{\mathfrak{C}}} g \circ f$. We claim that there is no loss of generality in assuming that the $\mathfrak{C}$-homotopy $F$ is constant, that is, $f \circ g=\mathrm{Id}_{\mathbb{Y}}$. Indeed, the $\mathfrak{C}$-homotopy $F$ partially lifts with

\footnotetext{
${ }^{6}$ If $\mathfrak{C}=$ EQUIV-TOP, we get the notion of an Equiv ${ }_{\mathcal{F}}$-soft map. If $\mathfrak{C}=$ ISOV-TOP, we get the notion of an $\operatorname{Isov}_{\mathcal{F}}$-soft map.
} 
respect to $f: f \circ g=F_{0}$. Since $f$ is a Hurewicz $\mathfrak{C}$-bundle, $F$ lifts to a $\mathfrak{C}$-homotopy $\widetilde{F}: \mathbb{Y} \times[0,1] \rightarrow \mathbb{X}$ with respect to $f$ such that $\widetilde{F}_{0}=g$. Clearly, $f \circ g^{\prime}=\operatorname{Id}_{\mathbb{Y}}$ and $g^{\prime} \circ f \simeq_{\mathfrak{C}} \widetilde{F}_{0} \circ f=g \circ f \simeq_{\mathfrak{C}} \operatorname{Id}_{\mathbb{X}}$ for the $\mathfrak{C}$-map $g^{\prime}=\widetilde{F}_{1}$.

Therefore we can assume without loss of generality that $f \circ g=\operatorname{Id}_{\mathbb{Y}}$ and $\operatorname{Id}_{\mathbb{X}} \stackrel{H}{\simeq} \mathfrak{C} g \circ f$. We consider the $\mathfrak{C}$-homotopy

$$
G \rightleftharpoons H \cup\left(g \circ f \circ H^{-1}\right): \mathbb{X} \times[0,2] \rightarrow \mathbb{X}
$$

connecting $\operatorname{Id}_{\mathbb{X}}$ with $g \circ f$ and define a constant $\mathfrak{C}^{2}$-homotopy $K: \mathbb{X} \times[0,2] \rightarrow \mathbb{Y}$ by the formula $K_{t}=f$ for all $t \in[0,2]$.

Since $f \circ H=f \circ(g \circ f \circ H)$, we easily see that $f \circ G: \mathbb{X} \times[0,2] \rightarrow \mathbb{Y}$ and $K: \mathbb{X} \times[0,2] \rightarrow \mathbb{Y}$ are connected by a $\mathfrak{C}$-homotopy which is constant on $\mathbb{A}=$ $\mathbb{X} \times\{0,2\}$. Since $f$ is a Hurewicz $\mathfrak{C}$-bundle, there is a $\mathfrak{C}$-homotopy $T: \mathbb{X} \times[0,2] \rightarrow \mathbb{X}$ such that $T_{0}=G_{0}=\operatorname{Id}_{\mathbb{X}}, T_{2}=G_{2}=g \circ f$ and $f \circ T=f$. Thus $T$ is fibrewise $\mathfrak{C}$-contractible.

Lemma 4.5. If $f: \mathbb{X} \rightarrow \mathbb{Y}$ is a fibrewise $\mathfrak{C}$-contractible Hurewicz $\mathfrak{C}$-bundle, then $f$ is $\mathfrak{C}$-soft.

Proof. Consider a commutative square $\mathcal{D}$ in the category $\mathfrak{C}$ and let $H: \mathbb{X} \times I \rightarrow \mathbb{X}$ be a $\mathfrak{C}$-homotopy such that $H_{1}=\mathrm{Id}, f \circ H_{t}=f$ and $H_{0}=s \circ f$ for some $\mathfrak{C}$-section $s: \mathbb{Y} \rightarrow \mathbb{X}$. Then we define $\mathfrak{C}$-maps $\Phi: \mathbb{A} \times[0,1] \cup \mathbb{Z} \times\{0\} \rightarrow \mathbb{X}$ and $\Psi: \mathbb{Z} \times[0,1] \rightarrow \mathbb{Y}$ by the formulae $\Phi(a, t)=H(\varphi(a), t), \Phi(z, 0)=s \circ \psi(z)$ and $\Psi(z, t)=\psi(z)$. Since $f \circ \Phi=\Psi \uparrow_{\mathbb{A} \times[0,1] \cup \mathbb{Z} \times\{0\}}$, by hypothesis there is a $\mathfrak{C}$-map $\widehat{\Phi}: \mathbb{Z} \times[0,1] \rightarrow \mathbb{X}$ extending $\Phi$ and satisfying $f \circ \widehat{\Phi}=\Psi$. Clearly, $\widehat{\Phi}_{1}$ is the desired $\mathfrak{C}$-extension of $\varphi$.

We say that a $G$-map $f: \mathbb{X} \rightarrow \mathbb{Y}$ is weakly Equiv $_{\mathcal{F}^{-}}$-soft if the map $f^{H}: \mathbb{X}^{H} \rightarrow \mathbb{Y}^{H}$ is soft for every $(H) \in \mathcal{F}$ with $\mathbb{Y}^{H} \neq \varnothing$.

Proposition 4.6. If $f: \mathbb{X} \rightarrow \mathbb{Y}$ is an Equiv $_{\mathcal{F}}$-soft $G$-map, then $f$ is weakly Equiv $_{\mathcal{F}}$-soft.

Remark 4.7. One can similarly prove that every $\operatorname{Isov}_{\mathcal{F}}$-soft map $f: \mathbb{X} \rightarrow \mathbb{Y}$ is weakly $\operatorname{IsOV}_{\mathcal{F}}$-soft.

Proof of Proposition 4.6. For every partial map $W \hookleftarrow A \stackrel{\varphi}{\longrightarrow} \mathbb{X}^{H},(H) \in \mathcal{F}$, we define a partial $G$-map $G / H \times W \hookleftarrow G / H \times A \stackrel{\Phi}{\rightarrow} \mathbb{X}$ by the formula $\Phi(g H, a)=g \cdot a$. Assume that $f \circ \varphi$ admits an extension $\psi: W \rightarrow \mathbb{Y}^{H}$. Then $\Theta \rightleftharpoons f \circ \Phi: G / H \times A \rightarrow \mathbb{Y}$ admits a $G$-extension $\Psi: G / H \times W \rightarrow \mathbb{Y}$ by the for-

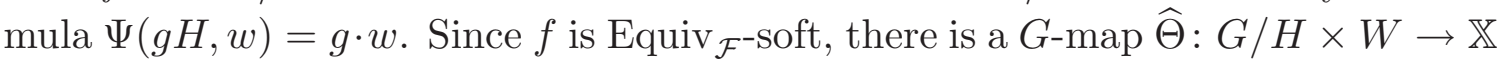
that extends $\Theta$ and covers $\Psi$. The restriction of $\widehat{\Theta}$ to $(e \cdot H) \times W$ is easily seen to be the desired extension of $\varphi$.

Let us examine the interrelation between the soft morphisms in different categories.

Theorem 4.8. Let $f: \mathbb{X} \rightarrow \mathbb{Y}$ be an isovariant map. If $f$ is (locally) Isov-soft, then it is (locally) Equiv-soft. 
Proof. Consider a commutative square

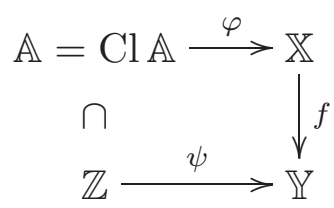

in the category EQUIV-TOP. Clearly, the formula ' $z_{1} \approx z_{2}$ if and only if $z_{1}, z_{2}$ lie on the same orbit and $\psi\left(z_{1}\right)=\psi\left(z_{2}\right)^{\prime}$ determines an equivalence relation on $\mathbb{Z}$. The natural map $h: \mathbb{Z} \rightarrow \mathbb{Z}^{\prime} \rightleftharpoons \mathbb{Z} / \approx$ is a perfect $G$-map and induces the identity map of the orbit spaces. Hence $\mathbb{Z}^{\prime}$ is a metric $G$-space, and $\mathbb{A}^{\prime}=\mathbb{A} / \approx$ is a closed $G$-subspace of $\mathbb{Z}^{\prime}$.

We easily see that the $G$-maps $\varphi$ and $\psi$ factor through isovariant maps $\varphi^{\prime}: \mathbb{A}^{\prime} \rightarrow \mathbb{X}$ and $\psi^{\prime}: \mathbb{Z}^{\prime} \rightarrow \mathbb{Y}$, that is, $\varphi=\varphi^{\prime} \circ h$ and $\psi=\psi^{\prime} \circ h$. If $f$ is Isov-soft, then we get a commutative square

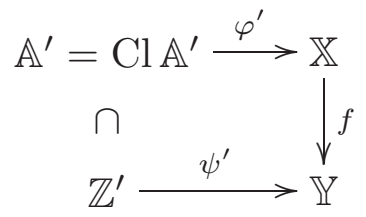

in the category ISOV-TOP. It admits an isovariant splitting $\widehat{\varphi}^{\prime}: \mathbb{Z}^{\prime} \rightarrow \mathbb{X}$. We easily see that $\widehat{\varphi}^{\prime} \circ h: \mathbb{Z} \rightarrow \mathbb{X}$ is the desired $G$-splitting of the commutative square (4.1).

The local variant of the theorem is proved in a similar way.

Clearly, the restriction of an Isov-soft map to the pre-image of an invariant subset is again Isov-soft. The following two results are concerned with the reverse situation.

Theorem 4.9. Let $f: \mathbb{X} \rightarrow \mathbb{Y}$ be an isovariant map. If $f$ is locally Isov-soft and $f_{G}: \mathbb{X}_{G} \rightarrow \mathbb{Y}_{G}$ and $g \rightleftharpoons f \uparrow: \mathbb{X} \backslash \mathbb{X}_{G} \rightarrow \mathbb{Y} \backslash \mathbb{Y}_{\mathbb{G}}$ are Isov-soft, then $f$ is Isov-soft. Proof. Since $\mathbb{X}$ admits a closed $G$-embedding $i: \mathbb{X} \hookrightarrow \mathbb{W}$ in an Isov-AE-space $\mathbb{W}$ (Theorem 3.2), $f: \mathbb{X} \rightarrow \mathbb{Y}$ is the restriction of the isovariant projection

$$
\varepsilon: \mathbb{Z} \rightleftharpoons\left\{(y, w) \in \mathbb{Y} \times \mathbb{W} \mid G_{y}<G_{w}\right\} \rightarrow \mathbb{Y}, \quad \varepsilon(y, w)=y,
$$

to the image of $\mathbb{X}$ under the diagonal embedding $\mathbb{X} \hookrightarrow \mathbb{Z}, x \mapsto(f(x), i(x))$.

The isovariant map $\varepsilon$ is Isov-soft by Theorem 6.1 (see below). To show that $f$ is Isov-soft, it suffices to prove the existence of a fibrewise isovariant retraction $R: \mathbb{Z} \rightarrow \mathbb{X}$ (that is, $\varepsilon=f \circ R$ ). Since $f_{G}$ is Isov-soft and $\mathbb{X}_{G} \subset \mathbb{Z}_{G}$ is closed, we can assume without loss of generality that $\mathbb{X}_{G}$ coincides with $\mathbb{Z}_{G}$. Since $f$ is locally Isov-soft, we can also assume that there is a neighbourhood fibrewise isovariant retraction $r: \mathbb{U} \rightarrow \mathbb{X}$. Therefore $r(\operatorname{Bd}(\mathbb{U})) \subset \mathbb{X} \backslash \mathbb{X}_{G}$.

Since $g=\left.f\right|_{\mathbb{X} \backslash \mathbb{X}_{G}}$ is Isov-soft, the commutative diagram

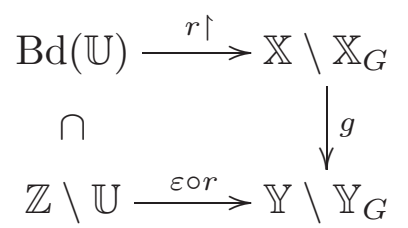


splits. This enables us to construct a fibrewise isovariant extension $r_{1}: \mathbb{Z} \backslash \mathbb{U} \rightarrow$ $\mathbb{X} \backslash \mathbb{X}_{\mathbb{G}}$. The maps $r$ and $r_{1}$ together determine the desired fibrewise isovariant retraction $R: \mathbb{Z} \rightarrow \mathbb{X}, R=r$ on $\mathbb{U}$ and $R=r_{1}$ on $\mathbb{Z} \backslash \mathbb{U}$.

The following theorem is obtained by an easy modification of the argument in [2], $\S 3.1$. We omit the proof.

Theorem 4.10. An isovariant map $f: \mathbb{X} \rightarrow \mathbb{Y}$ is Isov-soft in each of the following cases.

1) There is an infinite increasing sequence $\mathbb{Y}_{1} \subset \mathbb{Y}_{2} \subset \mathbb{Y}_{3} \subset \cdots, \mathbb{Y}_{n} \subset$ Int $\mathbb{Y}_{n+1}$, of closed $G$-subsets of $\mathbb{Y}$ such that $f$ is Isov-soft over each $\mathbb{Y}_{n}$.

2) There is an open $G$-covering $\left\{\mathbb{U}_{\lambda}\right\} \in \operatorname{cov}(\mathbb{Y})$ such that $f$ is Isov-soft over each $\mathbb{U}_{\lambda}$.

\section{$\S 5$. Isovariant extensors and twisted products}

We consider a compact group $G$ and a metric $H$-space $\mathbb{S}$, where $H<G$. The diagonal action of $H$ on the product $G \times \mathbb{S}$ is defined by the formula

$$
h \cdot(g, y) \rightleftharpoons\left(g \cdot h^{-1}, h \cdot y\right) .
$$

This action is free. Let $[g, y]$ stand for the element

$$
H \cdot(g, y)=\left\{\left(g \cdot h^{-1}, h \cdot y\right) \mid h \in H\right\}
$$

of the orbit space $(G \times \mathbb{S}) / H$. It turns out that the formula $g_{1} \cdot[g, y]=\left[g_{1} \cdot g, y\right]$, where $g, g_{1} \in G, y \in \mathbb{S}$, determines a well-defined continuous action of the whole group $G$ on the orbit space $(G \times \mathbb{S}) / H$, which is called the twisted product and is denoted by $G \times_{H} \mathbb{S}$.

Any $G$-map $\alpha: \mathbb{X} \rightarrow G / H$ to a homogeneous space with $H<G$ is called a slice map of $\mathbb{X}$. It is known that each orbit $G(x) \subset \mathbb{X}$ has a neighbourhood $\mathbb{U}$ admitting a slice map $\varphi: \mathbb{U} \rightarrow G / G_{x} \cong_{G} G(x)$ which is equal to the identity on this orbit [20]. The notion of a twisted product arises naturally in the study of $G$-spaces with slice maps because the following conditions are equivalent:

1) $\mathbb{X} \cong_{G} G \times_{H} \mathbb{S}$;

2) there is a slice map $\varphi: \mathbb{X} \rightarrow G / H, \varphi^{-1}([H])=\mathbb{S}$.

Other properties of twisted products (including their functorial properties) are described in detail, for example, in [20].

We say that an isovariant map $f: \mathbb{X} \rightarrow \mathbb{Y}$ admits a (non-trivial) tube structure if there are slice maps $\varphi: \mathbb{X} \rightarrow G / H$ and $\psi: \mathbb{Y} \rightarrow G / H, H \neq G$, such that $\varphi=\psi \circ f$. In this case we consider the isovariant $H$-map

$$
g \rightleftharpoons f \uparrow: \mathbb{S} \rightleftharpoons \varphi^{-1}[H] \rightarrow \mathbb{T} \rightleftharpoons \psi^{-1}[H] .
$$

Then $f$ can be represented in terms of the twisted product as follows:

$$
f=\operatorname{Id} \times f^{\prime}: G \times_{H} \mathbb{S}=\mathbb{X} \rightarrow G \times_{H} \mathbb{T}=\mathbb{Y}, \quad[g, s] \mapsto\left[g, f^{\prime}(s)\right]=[g, f(s)] .
$$

We now study the interrelation between the softness properties of $f$ and $g$. 
Theorem 5.1. If $f$ is (locally) Isov $_{G}$-soft, then $g$ is (locally) Isov $_{H}$-soft.

Proof. We only examine the case when $f$ is $\operatorname{Isov}_{G}$-soft. Consider a commutative square

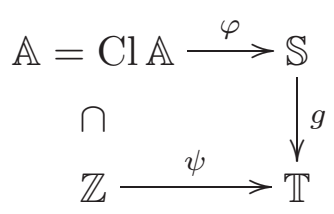

in the category $\mathrm{ISOV}_{H}$-TOP. The twisted product functor transforms it into the commutative square

$$
\begin{gathered}
G \times{ }_{H} \mathbb{A} \stackrel{\operatorname{Id} \times \varphi}{\longrightarrow} G \times{ }_{H} \mathbb{S} \\
\cap \\
G \times{ }_{H} \mathbb{Z} \stackrel{\text { Id } \times \psi}{\longrightarrow} G \times_{H} \mathbb{T}
\end{gathered}
$$

in the category ISOV $_{G}$-TOP. By hypothesis, one can $G$-split the commutative square (5.2) by a map $\widehat{\varphi}: G \times_{H} \mathbb{Z} \rightarrow G \times_{H} \mathbb{S}$. If $\widehat{\varphi}[e, z]=[g, s]$, where $e \in G$ is the identity, then we have $[e, \psi(z)]=[g, f(s)]$ since the diagram is commutative. Therefore $g \in H$ and $\widehat{\varphi}[e, z]=\left[e, g^{-1} s\right]$, that is, $\widehat{\varphi}(\mathbb{Z}) \subset \mathbb{S}$. Hence the restriction of $\widehat{\varphi}$ to $\mathbb{Z} \subset G \times{ }_{H} \mathbb{Z}$ is the desired $H$-splitting of the commutative square (5.1) into two triangular diagrams.

The local variant of the theorem is proved in a similar way.

Theorem 5.2. If $g$ is (locally) $\operatorname{Isov}_{H}$-soft, then $f$ is (locally) $\operatorname{Isov}_{G}$-soft.

Proof. We only examine the case when $g$ is Isov $_{H}$-soft. Consider a commutative square

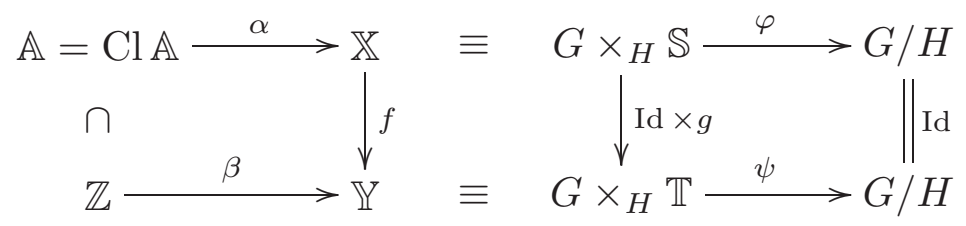

in the category ISOV $_{G^{-}}$TOP. Writing $\mathbb{Z}$ and $\mathbb{A}$ as $G \times_{H} \overline{\mathbb{Z}}$ and $G \times_{H} \overline{\mathbb{A}}$ respectively, where $\overline{\mathbb{A}} \rightleftharpoons(\varphi \circ \alpha)^{-1}([H])=\alpha^{-1}(\mathbb{S})$ and $\overline{\mathbb{Z}} \rightleftharpoons(\psi \circ \beta)^{-1}([H])=\beta^{-1}(\mathbb{T})$ are $H$-spaces, we naturally get a commutative square

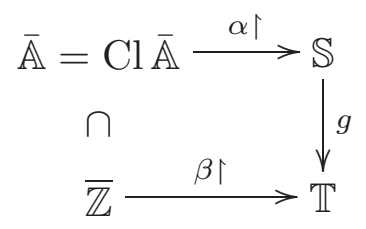

in the category ISOV $_{H}$-TOP. By hypothesis it admits an $H$-splitting $\chi: \overline{\mathbb{Z}} \rightarrow \mathbb{S}$. Applying the twisted product functor, we obtain the desired $G$-splitting $\operatorname{Id} \times_{H} \chi$ : $G \times_{H} \overline{\mathbb{Z}} \rightarrow G \times_{H} \mathbb{S}=\mathbb{X}$ of the commutative square (5.3).

The local variant of the theorem is proved in a similar way. 


\section{$\S 6$. The Borel construction}

We define a multi-valued map $\mathcal{E}: \mathbb{Y} \rightsquigarrow \mathbb{X}$ by the formula $\mathcal{E}(y)=\mathbb{X}^{G_{y}}$. Its graph $E(\mathbb{Y}, \mathbb{X}) \rightleftharpoons\left\{(y, x) \mid G_{y} \subset G_{x}\right\}$ is an invariant subset of $\mathbb{Y} \times \mathbb{X}$. Since $G_{(y, x)}=G_{y} \cap G_{x}=G_{y}$, the natural projection $\varepsilon: E(\mathbb{Y}, \mathbb{X}) \rightarrow \mathbb{Y}$ is an isovariant map.

Theorem 6.1. The space $\mathbb{X}$ is Equiv-AE (resp. Equiv-ANE) if and only if the isovariant map $\varepsilon: E(\mathbb{Y}, \mathbb{X}) \rightarrow \mathbb{Y}$ is isovariantly soft (resp. isovariantly locally soft) for every $G$-space $\mathbb{Y}$.

Remark 6.2. One can similarly prove that if $\mathbb{X} \in$ Equiv $_{\mathcal{F}}$-ANE, then the map

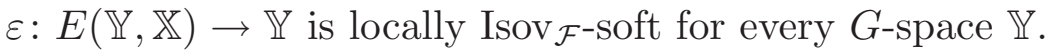

Proof of Theorem 6.1. To prove the necessity, we consider an admissible commutative diagram

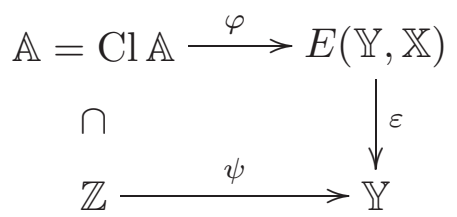

in the isovariant category. Since $\mathbb{X} \in$ Equiv-AE, there is an equivariant extension $\varphi^{\prime}: \mathbb{Z} \rightarrow \mathbb{X}$ coinciding with $\operatorname{pr}_{\mathbb{X}} \circ \varphi$ on $\mathbb{A}$, where $\operatorname{pr}_{\mathbb{X}}: E(\mathbb{Y}, \mathbb{X}) \rightarrow \mathbb{X}$ is the natural projection. We easily see that $\psi \times \varphi^{\prime}$ is the desired map $\widehat{\varphi}$. The sufficiency is not used in this paper, and therefore we omit its proof (hint: apply Theorem 1.1).

Applying Theorems 6.1, 4.8 in turn to an Equiv-ANE-space $\mathbb{X}$, we see that the $\operatorname{map} \varepsilon: E(\mathbb{Y}, \mathbb{X}) \rightarrow \mathbb{Y}$ is isovariantly locally soft and also locally Equiv-soft.

Suppose that $\mathcal{F} \subset \operatorname{Conj}_{G}$. In what follows, an Isov $\mathcal{F}-\mathrm{A}[\mathrm{N}] \mathrm{E}$-space will mean a $G$-F-space $\mathbb{X}$ such that every partial $G$-map $\mathbb{Z} \hookleftarrow \mathbb{A} \stackrel{\varphi}{\longrightarrow} \mathbb{X}$ given on a closed subset of the $G$ - $\mathcal{F}$-space $\mathbb{Z}$ can be extended to the whole of $\mathbb{Z}$ [to a $G$-neighbourhood $\mathbb{U} \subset \mathbb{Z}$ of $\mathbb{A}]$ to a $G$-map isovariant on the complement of $\mathbb{A}$ (note that this definition differs somewhat from that given in $\S 2$ ). By Theorem 3.1 we have $\mathbb{W}_{\mathcal{F}} \rightleftharpoons\{w \in \mathbb{W} \mid$ $\left.\left(G_{w}\right) \in \mathcal{F}\right\} \in \operatorname{Isov}_{\mathcal{F}}$-AE for every Isov-AE-space $\mathbb{W}$.

Theorem 6.3. If $\mathbb{X} \in$ Equiv $_{\mathcal{F}}-\mathrm{A}[\mathrm{N}] \mathrm{E}$ and $\mathbb{Y} \in \operatorname{Isov}_{\mathcal{F}}-\mathrm{A}[\mathrm{N}] \mathrm{E}$, then $E(\mathbb{Y}, \mathbb{X}) \in$ $\operatorname{Isov}_{\mathcal{F}}-\mathrm{A}[\mathrm{N}] \mathrm{E}$.

Proof. Consider a partial $G$-map

$$
\mathbb{Z} \hookleftarrow \mathbb{A} \stackrel{\varphi}{\rightarrow} E(\mathbb{Y}, \mathbb{X}), \quad \operatorname{Orb}(\mathbb{Z}) \subset \mathcal{F} .
$$

If $\mathbb{Y} \in \operatorname{Isov}_{\mathcal{F}}$-AE, then $\varepsilon \circ \varphi$ admits a $G$-extension $\psi: \mathbb{Z} \rightarrow \mathbb{Y}$ isovariant on the complement $\mathbb{Z} \backslash \mathbb{A}$. If $\mathbb{X} \in$ Equiv $_{\mathcal{F}^{-}} \mathrm{AE}$, then there is a $G$-extension $\chi: \mathbb{Z} \rightarrow \mathbb{X}$ of the composite $\mathbb{A} \stackrel{\varepsilon}{\rightarrow} E(\mathbb{Y}, \mathbb{X}) \stackrel{\mathrm{pr}_{2}}{\rightarrow} \mathbb{X}$. Then the image of the diagonal $G$-map $\psi \times \chi: \mathbb{Z} \rightarrow \mathbb{Y} \times \mathbb{X}$ is contained in $E(\mathbb{Y}, \mathbb{X})$ and, therefore, $\psi \times \chi$ determines a $G$-extension of $\varphi$. The local variant of the theorem is proved in a similar way.

We fix a $G$-space $\mathbb{W} \in$ Isov-AE and a family $\mathcal{F} \subset \operatorname{Conj}_{G}$. They determine a Borel functor

$$
E_{\mathcal{F}}: \mathrm{EQUIV}-\mathrm{TOP} \rightarrow \mathrm{ISOV}_{\mathcal{F}}-\mathrm{TOP}, \quad E_{\mathcal{F}}(\mathbb{X}) \rightleftharpoons\left\{(w, x) \mid G_{w} \subset G_{x},\left(G_{w}\right) \in \mathcal{F}\right\}
$$


which sends every $G$-map $f: \mathbb{X} \rightarrow \mathbb{Y}$ to an isovariant map $E_{f}: E_{\mathcal{F}}(\mathbb{X}) \rightarrow E_{\mathcal{F}}(\mathbb{Y})$ defined by the formula $E_{f}(w, x)=(w, f(x))$. (Borel suggested a similar construction for the single-element family $\mathcal{F}=\{e\}$.) Since $\mathbb{W}_{\mathcal{F}} \in \mathrm{Isov}_{\mathcal{F}}$-AE and $\mathrm{E}_{\mathcal{F}}(\mathbb{X})=\mathrm{E}\left(\mathbb{W}_{\mathcal{F}}, \mathbb{X}\right)$, Theorem 6.3 yields the following assertion.

$(*) E_{\mathcal{F}}(\mathbb{X}) \in \operatorname{Isov}_{\mathcal{F}}$-ANE whenever $\mathbb{X} \in$ Equiv $_{\mathcal{F}}$-ANE.

Let us show that the natural projection $p: E_{\mathcal{F}}(\mathbb{X}) \rightarrow \mathbb{X}, p(w, x)=x$, is a weak $G$-homotopy equivalence under certain conditions.

Theorem 6.4. The map $p: E_{\mathcal{F}}(\mathbb{X}) \rightarrow \mathbb{X}$ is Equiv $_{\mathcal{F}}$-soft for every $G$-space $\mathbb{X}$.

Proof. Consider an admissible (for checking the Equiv $\mathcal{F}^{-}$-softness of $p$ ) commutative square

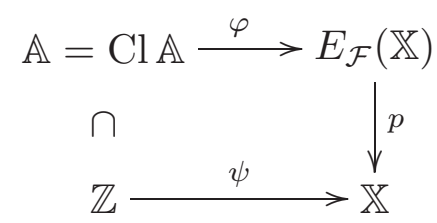

in the category EQUIV $\mathcal{F}^{-}$TOP. Since $\mathbb{W} \in$ Isov-AE, the partial map $\mathbb{Z} \hookleftarrow \mathbb{A} \stackrel{\chi}{\longrightarrow} \mathbb{W}$, where $\chi \rightleftharpoons \mathrm{pr}_{\mathbb{W}} \circ \varphi$ and $\mathrm{pr}_{\mathbb{W}}: E_{\mathcal{F}}(\mathbb{X}) \rightarrow \mathbb{W}$ is the natural projection, admits a $G$-extension $\widehat{\chi}: \mathbb{Z} \rightarrow \mathbb{W}$ isovariant on the complement. Therefore we have $G_{\widehat{\chi}(z)}=$ $G_{z} \subset G_{\psi(z)}$ and $\left(G_{\widehat{\chi}(z)}\right) \in \mathcal{F}$ for all $z \notin \mathbb{A}$. This enables us to verify that the formula $\widehat{\varphi}(z)=(\widehat{\chi}(z), \psi(z))$ yields the desired well-defined equivariant map $\widehat{\varphi}: \mathbb{Z} \rightarrow E_{\mathcal{F}}(\mathbb{X})$.

Combining this and Proposition 4.6, we get the following theorem.

Theorem 6.5. Let $\mathbb{X}$ be a $G$-space. Then the $G$-map $p: E_{\mathcal{F}}(\mathbb{X}) \rightarrow \mathbb{X}$ is:

1) a weak $G$-F-homotopy equivalence,

2) a $G$-homotopy equivalence provided that $\mathbb{X}$ is a $G$-F-space.

An embedding $e: X_{0} \hookrightarrow X$ is said to be homotopy dense if there is a homotopy $H: X \times I \rightarrow X$ such that $H_{0}=\operatorname{Id}$ and $\operatorname{Im} H_{t} \subset X_{0}$ for all $t>0$. The following assertion is easily verified.

(**) An embedding $e$ is homotopy dense if every partial map $Z \hookleftarrow A \stackrel{f}{\rightarrow} X$ can be extended to a map $\hat{f}: U \rightarrow X_{0}$ on a neighbourhood $U \supset A$ such that $\hat{f}(U \backslash A) \subset X_{0}$.

Clearly, every homotopy dense embedding is a homotopy equivalence.

Let $e$ be a homotopy dense embedding. Using the criterion for ANE-spaces in terms of small homotopies (see [22]), one can prove that $X_{0} \in \mathrm{ANE}$ if and only if $X \in \mathrm{ANE}$. The following result, which is an infinite-dimensional analogue of the so-called 'large gap' condition [19], highlights extensors in the isovariant category.

Theorem 6.6. Suppose that $\mathcal{F} \subset \operatorname{Conj}_{G}$ and $(H) \in \mathcal{F}$. If $\mathbb{X} \in$ Isov $_{\mathcal{F}}$-ANE, then the embedding $e: \mathbb{X}_{H} \hookrightarrow \mathbb{X}^{H}$ is homotopy dense.

Proof. Property $(* *)$ reduces the proof of the homotopy density of the embedding $\mathbb{X}_{H} \hookrightarrow \mathbb{X}^{H}$ to the extension of any partial map $Z \hookleftarrow A \stackrel{f}{\rightarrow} \mathbb{X}^{H}$ to a map $\hat{f}: U \rightarrow \mathbb{X}^{H}$ on a neighbourhood $U \supset A$ such that $\hat{f}(U \backslash A) \subset \mathbb{X}_{H}$.

Since $\mathbb{X} \in$ Isov $_{\mathcal{F}}$-ANE, the partial $G$-map $Z \times G / H \hookleftarrow A \times G / H \stackrel{\Phi}{\longrightarrow} \mathbb{X}$, $\Phi(a, g[H])=g f(a)$, can be $G$-extended to a $G$-map $\widehat{\Phi}: U \times G / H \rightarrow \mathbb{X}$ on 
a $G$-neighbourhood $U \times G / H \supset A \times G / H$ such that $\widehat{\Phi}$ is isovariant on $(U \backslash A) \times G / H$. Since the $G$-stabilizer of every point of $U \times e H$ coincides with $H$, it follows that the restriction $\widehat{\Phi}$ to $U \times e H$ is the desired extension of $f$.

The following proposition is proved in the same way as Theorem 6.6.

Proposition 6.7. Suppose that $\mathbb{X}$ is an $\operatorname{Isov}_{\mathcal{F}}$-AE-space and $(H) \in \mathcal{F}$. Then $\mathbb{X}_{H}$ is an absolute extensor and, therefore, is contractible.

\section{$\S 7$. The functor of isovariant gammafication}

In this section we use the method of reduction of isovariant homotopy problems to purely topological ones. To do this, we introduce the isovariant space of paths

$$
\mathbb{Y}_{\text {Isov }}^{I} \rightleftharpoons\left\{\lambda:[0,1] \rightarrow \mathbb{Y} \mid \operatorname{Im} \lambda \subset \mathbb{Y}_{H}, \text { where } H=G_{\lambda(0)}\right\} \subset \mathbb{Y}^{I}=C(I, \mathbb{Y})
$$

and define a $G$-map $p: \mathbb{Y}_{\text {Isov }}^{I} \rightarrow \mathbb{Y}$ by the formula $p(\lambda)=\lambda(0)$. Since the stabilizer of $\lambda \in \mathbb{Y}_{\text {Isov }}^{I}$ equals $\bigcap\left\{G_{\lambda(t)} \mid t \in I\right\}$ and therefore coincides with $G_{\lambda(0)}, p$ is an isovariant surjection.

The following result is an easy corollary of Theorem 2.1 .

Proposition 7.1. If $\mathbb{Y}$ is Isov $_{\mathcal{F}}-\mathrm{ANE}$, then $p$ is an Isov-soft map (and, therefore, $\left.\mathbb{Y}_{\text {Isov }}^{I} \in \operatorname{IsOv}_{\mathcal{F}}-\mathrm{ANE}\right)$.

Proof. Consider an admissible (for checking the $\operatorname{Isov}_{\mathcal{F}}$-softness of $p$ ) commutative square $\mathcal{D}$

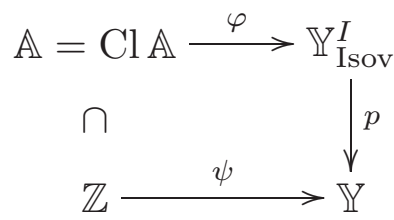

in the category ISOV-TOP. The isovariant map $\varphi$ is naturally represented as an isovariant map $\Phi: \mathbb{A} \times[0,1] \rightarrow \mathbb{Y}$. Together with $\psi$, it determines an isovariant map $\chi: \mathbb{Z} \times\{0\} \cup \mathbb{A} \times[0,1] \rightarrow \mathbb{Y}$. Since $\psi$ is isovariant, it follows that $\mathbb{Z}$ is a $G$ - $\mathcal{F}$-space. Since $\mathbb{Y} \in \operatorname{Isov}_{\mathcal{F}}$-ANE, $\chi$ admits an isovariant extension $\widehat{\chi}: \mathbb{Z} \times[0,1] \rightarrow \mathbb{Y}$ by Theorem 2.1. The resulting map $\hat{\chi}$ naturally induces an isovariant map $\widehat{\varphi}: \mathbb{Z} \rightarrow \mathbb{Y}_{\text {Isov }}^{I}$, which extends $\varphi$ and covers $\psi$.

Let $f: \mathbb{X} \rightarrow \mathbb{Y}$ be an isovariant map between Isov $_{\mathcal{F}}$-ANE-spaces $\mathbb{X}$ and $\mathbb{Y}$. Clearly, the fibrewise product $\Gamma_{f} \mathbb{X} \rightleftharpoons \mathbb{X}_{f} \times_{p} \mathbb{Y}_{\text {Isov }}^{I}$ coincides with $\left\{(x, \lambda) \in \mathbb{X} \times \mathbb{Y}_{\text {Isov }}^{I} \mid\right.$ $\lambda(0)=f(x)\}$. If there is no group action, then all constructions reduce to the cocylinder of a continuous map [24]. It follows from Proposition 7.1 that the map $\check{p}: \Gamma_{f} \mathbb{X} \rightarrow \mathbb{X}$ parallel to $p$ is isovariantly soft and, therefore, $\Gamma_{f} \mathbb{X} \in$ Isov $_{\mathcal{F}}$-ANE. It is straightforward to see that the space $\left(\Gamma_{f} \mathbb{X}\right)_{H}$ coincides with $\Gamma_{f_{H}}\left(\mathbb{X}_{H}\right)$ and the following assertion holds.

$(*)$ The maps $\left(\Gamma_{f}\right)_{H}:\left(\Gamma_{f} \mathbb{X}\right)_{H} \rightarrow \mathbb{Y}_{H}$ and $\Gamma_{f_{H}}: \Gamma_{f_{H}}\left(\mathbb{X}_{H}\right) \rightarrow \mathbb{Y}_{H}$ coincide.

We also define an isovariant map $\Gamma_{f}: \Gamma_{f} \mathbb{X} \rightarrow \mathbb{Y}$ by the formula $\Gamma_{f}(x, \lambda)=\lambda(1)$. One can regard $\Gamma_{f}$ as a functor on an appropriate category (the functor of isovariant gammafication). 
The isovariant map $\Gamma_{f}: \Gamma_{f} \mathbb{X} \rightarrow \mathbb{Y}$ is Isov-homotopy equivalent to the original isovariant map $f$. This can be deduced from the existence of an isovariant embedding $e: \mathbb{X} \hookrightarrow \Gamma_{f} \mathbb{X}, e(x)=\left(x, \delta_{f(x)}\right)$, where $\delta_{f(x)}$ is the constant path, along with an isovariant map $\theta: \Gamma_{f} \mathbb{X} \rightarrow \mathbb{X}, \theta(x, \lambda)=x$, which is Isov-homotopy inverse to $e$. Combining this with property $(*)$, we get the following proposition.

Proposition 7.2. The map $f$ is a (weak) Isov-homotopy equivalence if and only if $\Gamma_{f}: \Gamma_{f} \mathbb{X} \rightarrow \mathbb{Y}$ is a (weak) Isov-homotopy equivalence.

The isovariant map $\Gamma_{f}: \Gamma_{f} \mathbb{X} \rightarrow \mathbb{Y}$ is usually simpler than the isovariant map $f$. For example, it can be shown (but this will not be used here) that $\Gamma_{f}$ is a Hurewicz Isov $_{\mathcal{F}}$-bundle if $\mathbb{X}, \mathbb{Y} \in \operatorname{Isov}_{\mathcal{F}}$-ANE.

Theorem 7.3. Let $\mathbb{X}$ and $\mathbb{Y}$ be $\operatorname{Isov}_{\mathcal{F}}-\mathrm{A}[\mathrm{N}] \mathrm{E}$-spaces. Then the map $\Gamma_{f}: \Gamma_{f} \mathbb{X} \rightarrow \mathbb{Y}$ is (locally) Isov $\mathcal{F}$-soft.

Proof. Consider a commutative diagram

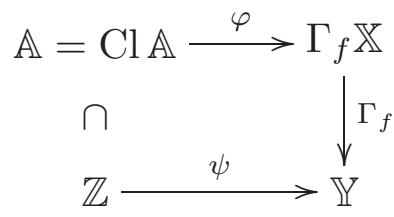

in the category $\operatorname{ISOV}_{\mathcal{F}}$-TOP. The map $\varphi: \mathbb{A} \rightarrow \Gamma_{f} \mathbb{X}$ is represented as the diagonal product $\left(\varphi_{1}, \varphi_{2}\right)$ of isovariant maps $\varphi_{1}: \mathbb{A} \rightarrow \mathbb{X}$ and $\varphi_{2}: \mathbb{A} \rightarrow \mathbb{Y}_{\text {Isov }}^{I}$. In its turn, $\varphi_{2}$ is naturally represented as an isovariant map $\Phi: \mathbb{A} \times[0,1] \rightarrow \mathbb{Y}$.

If $\mathbb{X} \in$ Isov $_{\mathcal{F}}-\mathrm{AE}$, then $\varphi_{1}$ admits an isovariant extension $\widehat{\varphi}_{1}: \mathbb{Z} \rightarrow \mathbb{X}$. The maps $f \circ \widehat{\varphi}_{1}, \Phi$ and $\psi$ together determine an isovariant map $\chi: \mathbb{Z} \times\{0\} \cup$ $\mathbb{A} \times[0,1] \cup \mathbb{Z} \times\{1\} \rightarrow \mathbb{Y}$. If $\mathbb{Y} \in \operatorname{Isov}_{\mathcal{F}}$-AE, then $\chi$ admits an isovariant extension $\hat{\chi}: \mathbb{Z} \times[0,1] \rightarrow \mathbb{Y}$. The resulting map $\widehat{\chi}$ naturally induces an isovariant map $\widehat{\varphi}_{2}: \mathbb{Z} \rightarrow \mathbb{Y}_{\text {Isov }}^{I}$. We easily see that the map $\left(\widehat{\varphi}_{1}, \widehat{\varphi}_{2}\right)$ is the desired map $\widehat{\varphi}: \mathbb{Z} \rightarrow \Gamma_{f} \mathbb{X}$.

The local variant of the theorem (when $\mathbb{X}, \mathbb{Y} \in$ Isov-ANE) is proved in a similar way.

The topological properties of $\Gamma_{f}$ can be further improved by imposing additional homotopy conditions on $f$. For example, the following important fact holds in the case of trivial group action (see [23] and [2]).

Theorem 7.4. Suppose that $X, Y \in$ ANE. If $f: X \rightarrow Y$ is a homotopy equivalence (not necessarily equivariant), then the map $\Gamma_{f}: \Gamma_{f} X \rightarrow Y$ is soft.

\section{$\S 8$. Proof of Theorem 1.1}

Theorem 1.1 has already been reduced to simpler assertions (see the introduction). We now proceed to prove them.

Reduction of Theorem 1.11 to Theorem 1.12. Consider an isovariant map $f: \mathbb{X} \rightarrow \mathbb{Y}$ between $\operatorname{Isov}_{\mathcal{F}}$-ANE-spaces $\mathbb{X}$ and $\mathbb{Y}$ which is a weak $\operatorname{Isov}_{\mathcal{F}}$-homotopy equivalence. By Proposition 7.2 and Theorem 4.3, to prove that $f$ is an Isov-homotopy equivalence, it suffices to establish that the map $\Gamma_{f}: \Gamma_{f} \mathbb{X} \rightarrow \mathbb{Y}$ is Isov-soft. 
Since $\Gamma_{f}$ is locally Isov-soft (Theorem 7.3), Theorem 1.12 yields the following assertion.

Lemma 8.1. If the map $\left(\Gamma_{f}\right)_{H}:\left(\Gamma_{f} \mathbb{X}\right)_{H} \rightarrow \mathbb{Y}_{H}$ is soft for every subgroup $H<G$, $(H) \in \mathcal{F}$, then $\Gamma_{f}$ is Isov-soft.

By hypothesis, the map $f_{H}: \mathbb{X}_{H} \rightarrow \mathbb{Y}_{H}$ is a homotopy equivalence of ANE-spaces. Hence Theorem 7.4 shows that the map $\Gamma_{f_{H}}: \Gamma_{f_{H}}\left(\mathbb{X}_{H}\right) \rightarrow \mathbb{Y}_{H}$ is a Hurewicz bundle and simultaneously a homotopy equivalence. Therefore it follows from Theorem 4.3 that the map $\Gamma_{f_{H}}$ is soft. However, the maps $\left(\Gamma_{f}\right)_{H}$ and $\Gamma_{f_{H}}$ coincide (see property $(*)$ in $\S 7)$ and, therefore, the map $\Gamma_{f}: \Gamma_{f} \mathbb{X} \rightarrow \mathbb{Y}$ is Isov-soft by Lemma 8.1.

Reduction of Theorem 1.8 to Theorem 1.12. Consider a commutative diagram

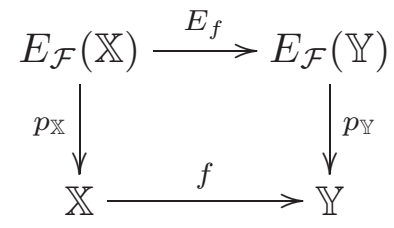

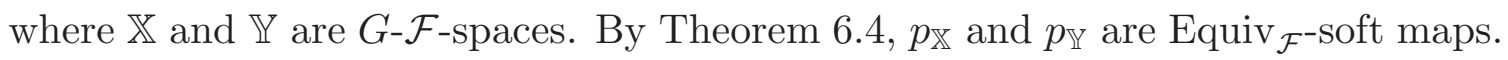

Proposition 8.2. The map $f$ is (locally) Equiv $_{\mathcal{F}^{-}}$soft if and only if the map $E_{f}$ is (locally) Isov-soft.

Proof. We consider the left commutative square

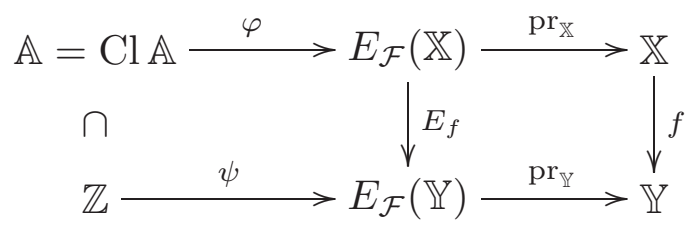

in ISOV-TOP and complement it with the right commutative square. Since $E_{\mathcal{F}}(\mathbb{Y})$ is a $G$ - $\mathcal{F}$-space and $\psi$ is isovariant, $\mathbb{Z}$ is also a $G$ - $\mathcal{F}$-space.

If $f$ is Equiv $_{\mathcal{F}^{-}}$-soft, then the $G$-map $\varphi_{2} \rightleftharpoons \operatorname{pr}_{\mathbb{X}} \circ \varphi$ extends equivariantly to a map $\widehat{\varphi}_{2}: \mathbb{Z} \rightarrow \mathbb{X}$ which is a lifting of the map $\psi_{2} \rightleftharpoons \operatorname{pr}_{\mathbb{Y}} \circ \psi$ with respect to $f$. Consider the diagonal product $\psi_{1} \times \widehat{\varphi}_{2}: \mathbb{Z} \rightarrow \mathbb{W} \times \mathbb{Y}$ of the isovariant map $\psi_{1} \rightleftharpoons$ $\operatorname{pr}_{\mathbb{W}} \circ \psi: \mathbb{Z} \rightarrow \mathbb{W}$ and $\widehat{\varphi}_{2}$. Clearly, it is an isovariant map sending $\mathbb{Z}$ to $E_{\mathcal{F}}(\mathbb{Y})$. Hence we get an isovariant map $\psi_{1} \times \widehat{\varphi}_{2}: \mathbb{Z} \rightarrow E_{\mathcal{F}}(\mathbb{Y})$ which extends $\varphi$ and is a lifting of $\psi$ with respect to $E_{f}$. The local variant of the necessity part is established in a similar way.

To establish the sufficiency, we consider the commutative square

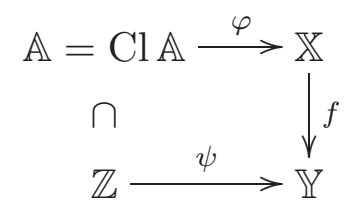

in the category EQUIV $\mathcal{F}^{-T O P . ~ C o n s i d e r ~ a ~} G$-lifting $\widetilde{\varphi}: \mathbb{A} \rightarrow E_{\mathcal{F}}(\mathbb{X})$ of $\varphi$ with

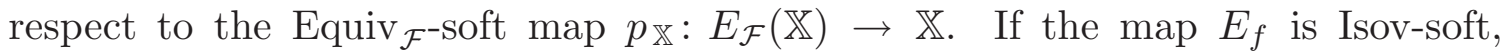
then it is Equiv-soft by Theorem 4.8, whence $f \circ p_{\mathbb{X}}=p_{\mathbb{Y}} \circ E_{f}$ is also Equiv-soft, 
being the composite of two Equiv-soft maps. Since the map $\widetilde{\varphi}$ is a partial $G$-lifting of $\psi$ with respect to $f \circ p_{\mathbb{X}}$, it follows that there is a $G$-lifting $\chi: \mathbb{Z} \rightarrow E_{\mathcal{F}}(\mathbb{X})$ of $\psi$ with respect to $f \circ p_{\mathbb{X}}$ and $\chi$ is an extension of $\widetilde{\varphi}$. The map $p_{\mathbb{X}} \circ \chi$ is the desired $G$-extension of $\varphi$ which lifts $\psi$ with respect to $f$. The local variant of the sufficiency part is established in a similar way.

Note that $\left(E_{f}(\mathbb{X})\right)_{H}=\mathbb{W}_{H} \times \mathbb{X}^{H}$ and $\left(E_{f}(\mathbb{Y})\right)_{H}=\mathbb{W}_{H} \times \mathbb{Y}^{H}$ while $\left(E_{f}\right)_{H}$ is naturally equivalent to the product $\mathrm{Id} \times f^{H}: \mathbb{W}_{H} \times \mathbb{X}^{H} \rightarrow \mathbb{W}_{H} \times \mathbb{Y}^{H}$. Therefore $\left(E_{f}\right)_{H}$ is soft if and only if $f^{H}$ is soft. It follows from Proposition 8.2 that Theorems 1.8, 1.12 are equivalent, but the latter is easier to prove.

Proof of Theorem 1.12. If $|G|=1$, then $f$ is a homeomorphism. Suppose that the theorem has already been proved for every proper subgroup $H<G$. We claim that it holds for every isovariant $G$-map $f: \mathbb{X} \rightarrow \mathbb{Y}$. By the following lemma it suffices to consider $G$-spaces $\mathbb{X}$ without $G$-fixed points.

Lemma 8.3. If Theorem 1.12 holds for all locally Isov-soft and simultaneously weakly Isov-soft maps $f: \mathbb{X} \rightarrow \mathbb{Y}$ of $G$-spaces without $G$-fixed points, then it also holds for all locally Isov-soft and simultaneously weakly Isov-soft maps $f$.

Proof. If Theorem 1.12 holds for the map $f \uparrow: \mathbb{X} \backslash \mathbb{X}_{G} \rightarrow \mathbb{Y} \backslash \mathbb{Y}_{G}$, then this map is Isov-soft. Since $f_{G}: \mathbb{X}_{G} \rightarrow \mathbb{Y}_{G}$ is also Isov-soft, the lemma follows from Theorem 4.9 .

We first consider the case when $f$ has non-trivial tube structure, that is, there are slice maps $\psi: \mathbb{Y} \rightarrow G / H, H \neq G$, and $\varphi=\psi \circ f: \mathbb{X} \rightarrow G / H$.

Lemma 8.4. If $f: \mathbb{X} \rightarrow \mathbb{Y}$ is an isovariant $G$-map with non-trivial tube structure, then Theorem 1.12 holds for $f$.

Proof. Consider the isovariant $H$-map $g \rightleftharpoons f \uparrow: \mathbb{S} \rightleftharpoons \varphi^{-1}[H] \rightarrow \mathbb{T} \rightleftharpoons \psi^{-1}[H]$. By Theorem 5.1, the $H$-isovariant map $g$ between $H$-spaces is locally $\mathrm{Isov}_{H}$-soft. It is also easy to see that $g_{K}: \mathbb{S}_{K} \rightarrow \mathbb{T}_{K}$ is soft for every $\mathbb{T}_{K} \neq \varnothing, K<H$, that is, $g$ is weakly Isov $_{H}$-soft. Since $H<G$ is a proper subgroup, $g$ is $\operatorname{Isov}_{H}$-soft by the inductive assumption. Then $f$ is Isov-soft by Theorem 5.2.

We now turn to the proof of the remaining case of the theorem (when $\mathbb{X}^{G}=$ $\left.\mathbb{Y}^{G}=\varnothing\right)$. By the Slice Theorem [20] it follows that every orbit $G(y) \subset \mathbb{Y}$ has an invariant neighbourhood with non-trivial tube structure. Hence the following lemma holds.

Lemma 8.5. There is an open $G$-covering $\left\{\mathbb{U}_{\lambda}\right\} \in \operatorname{cov}(\mathbb{Y})$ such that $f$ is an Isov-soft map over every $\mathbb{U}_{\lambda}$.

An application of Theorem 4.10, (2) completes the proof.

\section{$\S$ 9. Proofs of Theorems 1.3, 1.4, 1.6, 1.7}

Proof of Theorem 1.3. Since every $G-\mathcal{F}$-homotopy soft map is a weak $G$-homotopy equivalence, we need only prove the necessity part of the theorem. Consider a $G$-homotopy commutative diagram in the definition of the $G$ - $\mathcal{F}$-homotopy softness of $f: \mathbb{X} \rightarrow \mathbb{Y}$. The $G$-maps $p_{\mathbb{X}}: E_{\mathcal{F}}(\mathbb{X}) \rightarrow \mathbb{X}$ and $p_{\mathbb{Y}}: E_{\mathcal{F}}(\mathbb{Y}) \rightarrow \mathbb{Y}$ are $G$ - $\mathcal{F}$-soft 
by Theorem 6.4. Therefore $E_{\mathcal{F}}(\mathbb{X})$ and $E_{\mathcal{F}}(\mathbb{Y})$ are Equiv $\mathcal{F}_{-}$-ANE-spaces. Since $\operatorname{Orb}(\mathbb{Z}) \subset \mathcal{F}$, there are $G_{\text {-maps }} \widetilde{\varphi}: \mathbb{A} \rightarrow E_{\mathcal{F}}(\mathbb{X})$ and $\widetilde{\psi}: \mathbb{Z} \rightarrow E_{\mathcal{F}}(\mathbb{Y})$ such that $\varphi=p_{\mathbb{X}} \circ \widetilde{\varphi}$ and $\psi=p_{\mathbb{Y}} \circ \widetilde{\psi}$.

The $G$-maps $p_{\mathbb{X}}$ and $p_{\mathbb{Y}}$ are weak $G$ - $\mathcal{F}$-homotopy equivalences by Theorem 6.5 . Hence the map $E_{f}: E_{\mathcal{F}}(\mathbb{X}) \rightarrow E_{\mathcal{F}}(\mathbb{Y})$ is a weak $G$ - $\mathcal{F}$-homotopy equivalence of Equiv $_{\mathcal{F}}$-ANE-spaces and, consequently, it is a $G$-homotopy equivalence by Theorem 1.1. Therefore $E_{f}$ is a $G$-homotopy soft map (see property (*) in $\S 2$ ) and, consequently, there is a $G$-map $\chi: \mathbb{Z} \rightarrow E_{\mathcal{F}}(\mathbb{X})$ extending $\widetilde{\varphi}$ and satisfying $E_{f} \circ \chi \simeq_{G} \widetilde{\psi}$. We easily see that the map $p_{\mathbb{X}} \circ \chi$ is the desired $G$-extension $\widehat{\varphi}: \mathbb{Z} \rightarrow \mathbb{X}$ of $\varphi$.

Proof of Theorem 1.4. By Theorem 1.3 there is a $G$-map $h: \mathbb{X} \rightarrow \mathbb{Y}$ such that $g \circ h \simeq_{G} f$. Since the $G$-maps $f$ and $g$ are weak $G$ - $\mathcal{F}$-homotopy equivalences, the map $h$ is a weak $G$ - $\mathcal{F}$-homotopy equivalence of Equiv $\mathcal{F}_{\mathcal{F}}$-ANE-spaces. By Theorem $1.1, h$ is a $G$-homotopy equivalence.

Proof of Theorem 1.6. It suffices to consider an isovariant map $f: \mathbb{X} \rightarrow \mathbb{W}$ to an Isov-AE-space $\mathbb{W}$. Since $f(\mathbb{X}) \subset \mathbb{W}_{\mathcal{F}} \rightleftharpoons\left\{w \in \mathbb{W} \mid\left(G_{w}\right) \in \mathcal{F}\right\} \in$ Isov $_{\mathcal{F}}$-AE and $\mathbb{W}_{\mathcal{F}}^{H}$ is contractible for every subgroup $H<G$ with $(H) \in \mathcal{F}$ (Theorem 6.6), the map $f: \mathbb{X} \rightarrow \mathbb{W}_{\mathcal{F}}$ is a weak Equiv $\mathcal{F}$-homotopy equivalence.

Since Isov $_{\mathcal{F}}-\mathrm{ANE} \subset$ Equiv $_{\mathcal{F}}$-ANE, $\mathbb{W}_{\mathcal{F}} \in$ Equiv $_{\mathcal{F}^{-}}$ANE. Thus Theorem 1.1 applies and shows that the map $f: \mathbb{X} \rightarrow \mathbb{W}_{\mathcal{F}}$ is a $G$-homotopy equivalence, and the spaces $\mathbb{X} \in$ Equiv $_{\mathcal{F}}$-ANE and $\mathbb{W}_{\mathcal{F}} \in$ Equiv $_{\mathcal{F}^{-A N E}}$ have the same equivariant homotopy type. Using Proposition 2.2 , we obtain that $\mathbb{X} \in$ Equiv $_{\mathcal{F}}$ AE.

Proof of Theorem 1.7. Since the $G$-map $p: E_{\mathcal{C}}(\mathbb{X}) \rightarrow \mathbb{X}$ is Equiv $_{\mathcal{C}^{-}}$-soft by Theorem 6.4, it follows from Proposition 4.6 that $p^{H}:\left(E_{\mathcal{C}}(\mathbb{X})\right)^{H} \rightarrow \mathbb{X}^{H}$ is soft for all $(H) \in \mathcal{C}$. Since $\mathbb{X}^{H} \in \mathrm{AE}$ by hypothesis, it follows that $\left(E_{\mathcal{C}}(\mathbb{X})\right)^{H} \in \mathrm{AE}$ for all $(H) \in \mathcal{C}$. By Theorem 1.6 we have $E_{\mathcal{C}}(\mathbb{X}) \in$ Equiv $_{\mathcal{C}}$-AE.

Since $p$ is Equiv $_{\mathcal{C}}$-soft and $E_{\mathcal{C}}(\mathbb{X}) \in$ Equiv $_{\mathcal{C}}$-AE, we have a $G$-lifting $\psi: \mathbb{A} \rightarrow$ $E_{\mathcal{C}}(\mathbb{X})$ of $\varphi$ and a $G$-extension $\widehat{\psi}: \mathbb{Z} \rightarrow E_{\mathcal{C}}(\mathbb{X})$ of $\psi$. The map $p \circ \widehat{\psi}: \mathbb{Z} \rightarrow \mathbb{X}$ is the desired extension of $\varphi$.

\section{$\S 10$. Conclusion}

An important problem in equivariant algebraic topology is to find (calculate) the homotopy type of $\mathcal{F}$-classifying $G$-spaces. This problem is very difficult even for sufficiently small groups $G$ and non-trivial families $\mathcal{F}$. The effect of concentrating $\mathcal{F}$-classifying $G$-spaces (saying that each $\mathcal{F}$-orbit bundle $\mathbb{W}_{\mathcal{F}}$ of an Isov-AE-space $\mathbb{W}$ belongs to the class of Isov $_{\mathcal{F}}$-AE-spaces) provides additional possibilities for effective calculations (see [12]).

Another important problem is to detect isovariant extensors. In this paper we have obtained an Isov-AE-space as a product of appropriate $G$-spaces. However, such spaces arise from many other constructions in topology, geometry and analysis. Examples include the space $C(G, \mathbb{R})$ of the regular representation of $G$, the exponential space $\exp G$, the space of convex bodies in the Euclidean space $\mathbb{R}^{n}$, the space of linear isomorphisms of a Hilbert $G$-space, and many others. 
It would be interesting to prove Theorems 1.8 and 1.12 for arbitrary compact groups $G$. One might also develop the theory of shapes for the category ISOV-TOP and give a topological characterization of equivariant shape equivalences and equivariant fine homotopy equivalences in terms of the corresponding properties of maps of fixed-point sets. Moreover, there is the general problem of describing equivariant notions using topological properties of fixed-point sets; see [8], §8.1.

Let $f: X \rightarrow Y$ be locally soft. Then is it true that $f$ is soft if and only if $f \uparrow: f^{-1}(U) \rightarrow U$ is a homotopy equivalence for every open set $U \subset Y$ ?

The following question is interesting in connection with the Hilbert-Smith conjecture. Is it true that if the acting group $G$ is non-trivial and $\mathbb{X} \in$ Isov-AE, then $\operatorname{dim} X=\infty$ ?

\section{Bibliography}

[1] I. M. James and G. B. Segal, "On equivariant homotopy type", Topology 17:3 (1978), 267-272.

[2] I. M. James and G. B. Segal, "On equivariant homotopy theory", Topology Symposium (Siegen 1979), Lecture Notes in Math., vol. 788, Springer-Verlag, Berlin 1980, pp. 316-330.

[3] R. M. Seymour, "Some functional constructions on G-spaces", Bull. London Math. Soc. 15:4 (1983), 353-359.

[4] R. M. Seymour, "On G-cohomology theories and Künneth formulae", Current trends in algebraic topology, Part 1 (London, ON, 1981), CMS Conf. Proc., vol. 2, Amer. Math. Soc., Providence, RI 1982, pp. 251-271.

[5] Wu Yi Hsiang, Cohomology theory of topological transformation groups, Springer-Verlag, Berlin-Heidelberg-New York 1975; Russian transl., Mir, Moscow 1979.

[6] J. P. May, Equivariant homotopy and cohomology theory, CBMS Regional Conf. Ser. in Math., vol. 91, Amer. Math. Soc., Providence, RI 1996.

[7] T. tom Dieck, Transformation groups, de Gruyter Stud. Math., vol. 8, de Gruyter, Berlin-New York 1987.

[8] T. tom Dieck, Transformation groups and representation theory, Lecture Notes in Math., vol. 766, Springer-Verlag, Berlin 1979; Russian transl., Mir, Moscow 1982.

[9] G. E. Bredon, Equivariant cohomology theories, Lecture Notes in Math., vol. 34, Springer-Verlag, Berlin-New York 1967.

[10] S. Waner, "A generalization of the cohomology of groups", Proc. Amer. Math. Soc. 85:3 (1982), 469-474.

[11] S. Waner, "Mackey functors and G-cohomology", Proc. Amer. Math. Soc. 90:4 (1984), 641-648.

[12] S. M. Ageev, "On Palais universal $G$-spaces and isovariant absolute extensors", Mat. Sb. 203:6 (2012), 3-34; English transl., Sb. Math. 203:6 (2012), 769-797.

[13] M. Murayama, "On G-ANR's and their G-homotopy types", Osaka J. Math. 20:3 (1983), 479-512.

[14] R. S. Palais, "The classification of $G$-spaces", Mem. Amer. Math. Soc., vol. 36, Amer. Math. Soc., Providence, RI 1960.

[15] S. M. Ageev and D. Repovsh, "On the problem of extending the lifting homotopy for compact transformation groups", Mat. Zametki (to appear). (Russian) 
[16] S. M. Ageev, "Free equivariant extensors", General topology. Spaces and maps, Moscow Univ. Press, Moscow 1994, pp. 2-8.

[17] S. M. Ageev, "Classification of G-spaces", Izv. Ross. Akad. Nauk Ser. Mat. 56:6 (1992), 1345-1357; English transl., Russian Acad. Sci. Izv. Math. 41:3 (1993), $581-591$.

[18] S. M. Ageev and D. Repovsh, "On extending actions of groups", Mat. Sb. 201:2 (2010), 3-28; English transl., Sb. Math. 201:2 (2010), 159-182.

[19] S. Weinberger, The topological classification of stratified spaces, Chicago Lectures in Math., Chicago State Univ. Press, Chicago, IL 1994.

[20] G. E. Bredon, Introduction to compact transformation groups, vol. 46, Academic Press, New York-London 1972; Russian transl., Nauka, Moscow 1980.

[21] K. Borsuk, Theory of retracts, Państwowe Wydawnictwo Naukowe, Warsaw 1967; Russian transl., Mir, Moscow 1971.

[22] S.-T. Hu, Theory of retracts, Wayne State Univ. Press, Detroit 1965.

[23] T. tom Dieck, K. H. Kamps, and D. Puppe, Homotopietheorie, Lecture Notes in Math., vol. 157, Springer-Verlag, Berlin-New York 1970.

[24] M. M. Postnikov, Lectures on algebraic topology. Foundations of homotopy theory, Nauka, Moscow 1984. (Russian)

S. M. Ageev

Belarus State University, Minsk

E-mail: ageev_sergei@yahoo.com
Received 15/NOV/10

$14 / \mathrm{NOV} / 11$

Translated by THE AUTHOR 